\title{
EXISTENCE RESULTS TO A CLASS OF NONLINEAR PARABOLIC SYSTEMS INVOLVING POTENTIAL AND GRADIENT TERMS
}

\author{
B. ABDELlAOUI, A. ATTAR, R. BENTIFOUR, E.-H. LAAMRI
}

\begin{abstract}
In this paper, we investigate the existence of solutions to a nonlinear parabolic system, which couples a non-homogeneous reaction-diffusion-type equation and a non-homogeneous viscous Hamilton-Jacobi one. The initial data and right-hand sides satisfy suitable integrability conditions and non-negative. In order to simplify the presentation of our results, we will consider separately two simplified models : first, vanishing initial data, and then, vanishing right-hand sides.
\end{abstract}

\section{INTRODUCTION}

The main goal of this paper is to study the existence of solutions to the following non-linear system

$$
\left\{\begin{array}{ccccc}
u_{t}-\Delta u & = & v^{q}+f & \text { in } & \Omega_{T}=\Omega \times(0, T), \\
v_{t}-\Delta v & = & \left.\nabla u\right|^{p}+g & \text { in } & \Omega_{T}=\Omega \times(0, T), \\
u & = & v=0 & \text { on } & \Gamma_{T}=\partial \Omega \times(0, T), \\
u(x, 0) & = & u_{0}(x) & \text { in } & \Omega, \\
v(x, 0) & = & v_{0}(x) & \text { in } & \Omega, \\
u, v & \geq & 0 & \text { in } & \Omega \times(0, T),
\end{array}\right.
$$

where $\Omega$ is a bounded domain of $\mathbb{R}^{N}, N \geq 1$ and $p, q \geq 1$. Here $(f, g)$ and $\left(u_{0}, v_{0}\right)$ are non-negative data and satisfy some suitable integrability conditions that we will specify later. Our objective is to find "natural" relation between $p, q$ and the regularity of the data in order to get the existence of a solution to system (1.1).

Systems with gradient term appear for instance when considering electrochemical models in engineering and some other models in fluid dynamics. We refer to [23] and [28] for more details and more applications of this class of systems.

Before stating our main results, let us begin by recalling some previous results related to our system.

Stationary case: Concerning existence of solutions, it is well known in some particular cases. For this, we refer to [19], [20], [8] and [1] where some general existence results were established.

Parabolic case: In the case of a single equation and under the presence of gradient term, many results of global existence are known. We refer to $[2,18]$ and the references therein. On the other

Date: Version révisée le 03 décembre 2019 et publiée dans MJM en 2020.

Key words and phrases. Parabolic System, nonlinear gradient terms, apriori estimates, Fixed point Theorem. 2000 Mathematics Subject Classification:MSC 2000:35B05, 35K15, 35B40, 35K55, 35K65.

The first author is partially supported by project MTM2016-80474-P, MINECO, Spain. 
hand, there is an extensive literature devoted to the study and solvability and properties of solutions to the so-called viscous Hamilton-Jacobi Equation $(H J)$.

More precisely, let us consider the following Dirichlet and Cauchy problems :

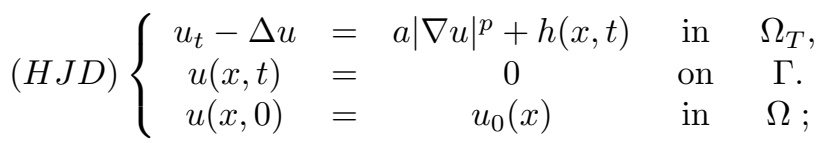

and

$$
(H J C)\left\{\begin{aligned}
u_{t}-\Delta u & =a|\nabla u|^{p}+h(x, t) & & \text { in } \mathbb{R}^{N} \times(0, T), \\
u(x, 0) & =u_{0}(x) & & \text { in } \mathbb{R}^{N},
\end{aligned}\right.
$$

where $a \in \mathbb{R}^{*}, p \geq 0$.

First case: If $h \equiv 0$. In bounded domains, existence and uniqueness results of (HJD) may be found for example in $[4,10,24,47,48]$ and the references therein. A considerable literature has also been devoted to the analogous whole-space Cauchy problem $(H J C)$; we refer to $[7,12,13$, $14,35,49]$ and the references therein.

Needless to say, the references mentioned above do not exhaust the rich literature on the subject. Second case: If $h \not \equiv 0$. As far as we know, Problems (HJD) and $(H J C)$ have been considered in $[50,51]$. We recall also the result of $[2]$ where, in the case $p=2$, sharp regularity results are proved for positive solution to problem $(H J D)$ and, as a consequence, a complete classification of the set of nonnegative solutions is obtained in relation with the classical parabolic capacity.

In the case of parabolic systems, we refer to [37], [36], [29], [32], [40], [44] and the references therein . Let us briefly recall some related results. The case where the gradient term appears as an absorption term was treated in [40]. A particular case with natural growth in the gradient was considered in [29]. We also mention the paper [16] where applications of a such parabolic systems are given in the context of stochastic differential games.

Systems with potential nonlinearities was studied in depth in [32], where the authors proved the existence of a Fujita-type exponents. An interesting contribution for this class of systems was recently made in [44, section 32$]$.

Our main contribution in this work is to get the existence of solution to (1.1) for all $p, q \geq 1$ under "natural" conditions on the data $\left(u_{0}, v_{0}\right)$ and $(f, g)$. By solution, we mean either energy solution or entropy solution (for more specifics, see Definition 2.2 and Definition 2.5).

In order to simplify the presentation, we will mostly discuss two simplified models (we refer to the proof of Theorem 3.3 for the relation between the two cases).

(1) The first one is obtained by taking $\left(u_{0}, v_{0}\right)=(0,0)$ in (1.1), namely:

$$
\left\{\begin{array}{ccccc}
u_{t} & = & \Delta u+v^{q}+f & \text { in } & \Omega_{T}, \\
v_{t} & = & \Delta v+|\nabla u|^{p}+g & \text { in } & \Omega_{T}, \\
u=v & = & 0 & \text { on } & \Gamma_{T}, \\
u(x, 0) & = & 0 & \text { in } & \Omega, \\
v(x, 0) & = & 0 & \text { in } & \Omega, \\
u, v & \geq & 0 & \text { in } & \Omega_{T} .
\end{array}\right.
$$

Using regularity arguments and a suitable fixed-point Theorem, we get the existence of a solution to (1.2) in a suitable parabolic-Sobolev space. 
(2) The second simplified model corresponds to $(f, g)=(0,0)$, i.e:

$$
\left\{\begin{array}{ccccc}
u_{t} & = & \Delta u+v^{q} & \text { in } & \Omega_{T}, \\
v_{t} & = & \Delta v+|\nabla u|^{p} & \text { in } & \Omega_{T}, \\
u=v & = & 0 & \text { on } & \Gamma_{T}, \\
u(x, 0) & = & u_{0}(x) & \text { in } & \Omega, \\
v(x, 0) & = & v_{0}(x) & \text { in } & \Omega, \\
u, v & \geq & 0 & \text { in } & \Omega_{T} .
\end{array}\right.
$$

Taking advantage from the study of the first model, and using a change of function, we will prove that system (1.3) has a solution under suitable hypotheses on initial data $\left(u_{0}, v_{0}\right)$.

In a forthcoming work, we shall investigate questions of global existence and Fujita type blow-up for the whole-space Cauchy problem.

The paper is composed of four sections. In Section 2 we give some useful tools like the notion of parabolic-Sobolev spaces and some of their properties. We also specify the sense in which solutions are considered, as well as some optimal regularity results. The first part of Section 3 is devoted to get existence results, and is split into two parts : Subsection 3.1 is devoted to the first simplified model (1.2) and Subsection 3.2 for the second one (1.3). In Subsection 3.3 we analyze the question of blow-up in finite time, in a suitable norm of the solution and under additional condition on $p, q$. The last section is devoted to treating variants of the previous model, where the gradient term appears in both evolution equations.

\section{Preliminaries Results and Functional Setting}

In this Section we give some useful tools that will be used many times in this paper.

2.1. Functional setting. Let $r \geq 1$. In the sequel we denote by $L^{r}\left(0, T ; W_{0}^{1, r}(\Omega)\right)$ the set of functions $u$ such that $u \in L^{r}\left(\Omega_{T}\right)$ and $u(., t) \in W_{0}^{1, r}(\Omega)$. The space $L^{r}\left(0, T ; W_{0}^{1, r}(\Omega)\right)$ is equipped with the norm

$$
\|u\|_{L^{r}\left(0, T ; W_{0}^{1, r}(\Omega)\right)}:=\left(\int_{0}^{T} \int_{\Omega}|\nabla u(x, t)|^{r} d x d t\right)^{\frac{1}{r}}
$$

is a Banach space. We shall often refer to this space by the shorthand $E_{r}\left(\Omega_{T}\right):=L^{r}\left(0, T ; W_{0}^{1, r}(\Omega)\right)$.

For $s, r \geq 1$, the space $V_{0}^{s, r}\left(\Omega_{T}\right):=L^{\infty}\left(0, T ; L^{s}(\Omega)\right) \cap E_{r}\left(\Omega_{T}\right)$ endowed with the norm

$$
\|\varphi\|_{V_{0}^{s, r}\left(\Omega_{T}\right)}:=\operatorname{ess} \sup _{0 \leq t \leq T}\|\varphi(., t)\|_{L^{s}(\Omega)}+\|\varphi\|_{E_{r}\left(\Omega_{T}\right)}
$$

is also a Banach space. If $s=1$, we have,

$$
V_{0}^{1, r}\left(\Omega_{T}\right)=L^{\infty}\left(0, T ; L^{1}(\Omega)\right) \cap E_{r}\left(\Omega_{T}\right) .
$$

The next proposition will be useful in order to show a priori estimates, and it will be used throughout this work. We refer to [31, Proposition 3.1] for more details.

Proposition 2.1. Let $s, r \geq 1$ and $\nu=r \frac{N+s}{N}$, then there exists a positive constant $C$ depending only on $N, \nu, s$ such that for all $v \in V_{0}^{s, r}\left(\Omega_{T}\right)$,

$$
\iint_{\Omega_{T}}|v(x, t)|^{\nu} d x d t \leq C^{\nu}\left(\iint_{\Omega_{T}}|\nabla v(x, t)|^{r} d x d t\right)\left(e s s \sup _{0 \leq t \leq T} \int_{\Omega}|v(x, t)|^{s} d x d t\right)^{\frac{r}{N}}
$$


moreover

$$
\|v\|_{L^{\nu}\left(\Omega_{T}\right)} \leq C\|v\|_{V_{0}^{s, r}\left(\Omega_{T}\right)} .
$$

The multiplicative inequality (2.1) and the embedding inequality (2.2) continue to hold for functions $v \in V^{s, r}\left(\Omega_{T}\right)$ such that

$$
\int_{\Omega} v(x, t) d x=0, \quad \text { for all a.e } t \in(0, T) .
$$

- Notions of solution : Now, we define the two notions of solution that we will systematically use throughout this paper : energy solution and entropy solution.

The starting point is to treat each equation in system (1.1) separately.

To this end, let us consider the problem

$$
\left\{\begin{array}{ccccc}
w_{t}-\Delta w & = & h & \text { in } & \Omega_{T}, \\
w & = & 0 & \text { on } & \Gamma_{T}, \\
w(x, 0) & = & w_{0}(x) & \text { in } & \Omega .
\end{array}\right.
$$

Definition 2.2. Assume $\left(h, w_{0}\right) \in L^{2}\left(0, T ; H^{-1}(\Omega)\right) \times L^{2}(\Omega)$. We say that $w$ is an energy solution to problem $(2.3)$ if $w \in L^{2}\left(0, T ; H_{0}^{1}(\Omega)\right) \cap \mathcal{C}\left(0, T ; L^{2}(\Omega)\right), w_{t} \in L^{2}\left(0, T ; H^{-1}(\Omega)\right), w(x, t) \rightarrow w_{0}$ strongly in $L^{2}(\Omega)$ as $t \rightarrow 0$ and for all $v \in L^{2}\left(0, T ; H_{0}^{1}(\Omega)\right)$ we have

$$
\int_{0}^{T}\left\langle w_{t}, v\right\rangle d t+\iint_{\Omega_{T}} \nabla w \cdot \nabla v d x d t=\int_{0}^{T}\langle h(x, t), v\rangle d t .
$$

Related to the nonlinear system (1.1), we have the following definition of energy solution.

Definition 2.3. (Energy solution) Assume that $\left(f, u_{0}\right),\left(g, v_{0}\right) \in L^{2}\left(0, T ; H^{-1}(\Omega)\right) \times L^{2}(\Omega)$. Let $(u, v) \in\left(L^{2}\left(0, T ; H_{0}^{1}(\Omega)\right) \cap \mathcal{C}\left(0, T ; L^{2}(\Omega)\right)\right)^{2}$ be nonnegative functions such that $\left(u_{t}, v_{t}\right) \in\left(L^{2}\left(0, T ; H^{-1}(\Omega)\right)\right)^{2}$. Define $F(x, t):=v^{q}+f, G(x, t):=|\nabla u|^{p}+g$.

We say that $(u, v)$ is an energy solution to the system (1.1) if $F, G \in L^{2}\left(0, T ; H^{-1}(\Omega)\right), u(\cdot, t) \rightarrow$ $w_{0}, v(\cdot, t) \rightarrow v_{0}$ strongly in $L^{2}(\Omega)$ as $t \rightarrow 0$ and for all $(\psi, \theta) \in\left(L^{2}\left(0, T ; H_{0}^{1}(\Omega)\right)\right)^{2}$, we have

$$
\begin{gathered}
\int_{0}^{T}\left\langle u_{t}, \psi\right\rangle d t+\iint_{\Omega_{T}} \nabla u \cdot \nabla \psi d x d t=\int_{0}^{T}\langle F(x, t), \psi\rangle d t, \\
\int_{0}^{T}\left\langle v_{t}, \theta\right\rangle d t+\iint_{\Omega_{T}} \nabla v \cdot \nabla \theta d x d t=\int_{0}^{T}\langle G(x, t), \theta\rangle d t .
\end{gathered}
$$

In the case of $L^{1}$ data, we need to use the concept of entropy solution introduced in [42] (which is equivalent, in this case, to the concept of renormalized solution defined in [17]). We note that the concept of entropy solution was introduced for the first time in [15] to treat nonlinear elliptic problem with general data.

Let us first recall the following definition:

Definition 2.4. Let $p \geq 1$ and let $w$ be a measurable function. We say that $w \in \mathcal{T}_{0}^{1, p}\left(\Omega_{T}\right)$ if $T_{k}(w) \in L^{p}\left(0, T ; W_{0}^{1, p}(\Omega)\right)$ for all $k>0$ where

$$
T_{k}(s):=\left\{\begin{array}{ccc}
s & \text { if } & |s| \leq k ; \\
k \frac{s}{|s|} & \text { if } & |s|>k .
\end{array}\right.
$$


We begin by stating the defining of the entropy solution in the case of scalar equation.

Definition 2.5. Assume that $\left(h, w_{0}\right) \in L^{1}\left(\Omega_{T}\right) \times L^{1}(\Omega)$. We say that $w \in \mathcal{C}\left(0, T ; L^{1}(\Omega)\right)$ is an entropy solution to problem $(2.3)$ if $w \in \mathcal{T}_{0}^{1,2}\left(\Omega_{T}\right)$ and for all $v \in L^{2}\left(0, T ; H_{0}^{1}(\Omega)\right) \cap L^{\infty}\left(\Omega_{T}\right) \cap$ $\mathcal{C}\left(0, T ; L^{1}(\Omega)\right)$ and for all $k>0$,

$$
\begin{aligned}
\int_{\Omega} \Theta_{k}(w-v)(T) d x & +\iint_{\Omega_{T}} v_{t} T_{k}(w-v) d x d t+\iint_{\Omega_{T}} \nabla w \nabla \Theta_{k}(w-v) d x d t \\
& =\int \Theta_{\Omega} \Theta_{k}\left(w_{0}-v(0, .)\right) d x+\iint_{\Omega_{T}} h T_{k}(w-v) d x d t
\end{aligned}
$$

where

$$
\Theta_{k}(s):=\int_{0}^{s} T_{k}(t) d t
$$

Notice that,

$$
\int_{\Omega} w_{t} T_{k}(w) d x=\frac{d}{d t}\left(\int_{\Omega} \Theta_{k}(w) d x\right)
$$

where

$$
\int_{\Omega} \Theta_{k}(w(x, t)) d x-\int_{\Omega} \Theta_{k}(w(x, 0)) d x=\int_{0}^{t} \int_{\Omega} w_{s} \Theta_{k}(w) d x d s .
$$

If $w$ is an entropy solution to problem (2.3), then $w$ is a distributional solution.

In a similar way, we define the entropy solution for system (1.1).

Definition 2.6. (Entropy solution) Assume that $\left(f, u_{0}\right),\left(g, v_{0}\right) \in L^{1}\left(\Omega_{T}\right) \times L^{1}(\Omega)$. Let $(u, v) \in$ $\left(\mathcal{C}\left(0, T ; L^{1}(\Omega)\right)\right)^{2}$ be nonnegative functions. We say that $(u, v)$ is an entropy solution to system (1.1) if $(u, v) \in\left(\mathcal{T}_{0}^{1,2}\left(\Omega_{T}\right)\right)^{2}, F, G \in L^{1}\left(\Omega_{T}\right)$ (where $F, G$ are defined as in Definition 2.3), and for all $(\psi, \theta) \in\left(L^{2}\left(0, T ; H_{0}^{1}(\Omega)\right) \cap L^{\infty}\left(\Omega_{T}\right) \cap \mathcal{C}\left(0, T ; L^{1}(\Omega)\right)\right)^{2}$ and for all $k>0$,

$$
\begin{aligned}
\int_{\Omega} \Theta_{k}(u-\psi)(T) d x & +\iint_{\Omega_{T}} \psi_{t} T_{k}(u-\psi) d x d t+\iint_{\Omega_{T}} \nabla u \nabla \Theta_{k}(u-\psi) d x d t \\
& =\int \Theta_{\Omega} \Theta_{k}\left(u_{0}-\psi(0, .)\right) d x+\iint_{\Omega_{T}} F T_{k}(u-\psi) d x d t \\
\int_{\Omega} \Theta_{k}(v-\theta)(T) d x & +\iint_{\Omega_{T}} \theta_{t} T_{k}(v-\theta) d x d t+\iint_{\Omega_{T}} \nabla v \nabla \Theta_{k}(u-\theta) d x d t \\
& =\iint_{\Omega} \Theta_{k}\left(u_{0}-\theta(0, .)\right) d x+\iint_{\Omega_{T}} G T_{k}(v-\theta) d x d t
\end{aligned}
$$

The next existence and compactness result is proved in [42] and [17].

Theorem 2.7. Let $\left(h, w_{0}\right) \in L^{1}\left(\Omega_{T}\right) \times L^{1}(\Omega)$. Then, the problem (2.3) has a unique entropy solution $w \in \mathcal{C}\left(0, T ; L^{1}(\Omega)\right)$ such that $w \in L^{s}\left(0, T ; W_{0}^{1, s}(\Omega)\right)$ for all $s<\frac{N+2}{N+1}$. Moreover, there exists a positive constant $C=C(\Omega, N$, s) such that

$$
\|w\|_{\mathcal{C}\left(0, T ; L^{1}(\Omega)\right)}+\|w\|_{L^{s}\left(0, T ; W_{0}^{1, s}(\Omega)\right)} \leq C\left(\left\|w_{0}\right\|_{L^{1}(\Omega)}+\|h\|_{L^{1}\left(\Omega_{T}\right)}\right) .
$$

As a consequence of Proposition 2.1, it holds that, for all $\nu<\frac{N+2}{N}$ and for all $r<\frac{N+2}{N+1}$,

$$
\iint_{\Omega_{T}}|w(x, t)|^{\nu} d x d t \leq C^{\nu}\left(\iint_{\Omega_{T}}|\nabla w(x, t)|^{r} d x d t\right)\left(e s s \sup _{0 \leq t \leq T} \int_{\Omega}|w(x, t)| d x d t\right)^{\frac{r}{N}} .
$$


Furthermore, for $s<\frac{N+2}{N+1}$ fixed, the operator $L:\left(h, w_{0}\right) \mapsto u$ is compact from $L^{1}\left(\Omega_{T}\right) \times L^{1}(\Omega)$ to $L^{s}\left(0, T ; W_{0}^{1, s}(\Omega)\right)$.

Now, let us recall some regularity results that will allow us to establish some a priori estimates when dealing with approximating problems. We refer to $[14,18,44]$ for a complete proof.

Theorem 2.8. Let $w$ be the unique solution (in sense of Definition 2.5) to problem (2.3) with $\left(h, w_{0}\right) \in L^{\kappa}\left(\Omega_{T}\right) \times L^{\tau}(\Omega)$. Then, we have

The case $w_{0} \equiv 0$ :

- If $1<\kappa<\frac{N+2}{2}$, then $w \in L^{\delta}\left(\Omega_{T}\right)$ where $\delta=\frac{(N+2) \kappa}{N+2-2 \kappa}, w \in E_{\bar{\kappa}}\left(\Omega_{T}\right)$ where $\bar{\kappa}=$ $\frac{(N+2) \kappa}{N+2-\kappa}$ and $w \in L^{\infty}\left(0, T ; L^{a}(\Omega)\right)$ where $a=\frac{\kappa N}{N+2-2 \kappa}$.

Moreover, there exists $C=C(\Omega, N, \kappa)>0$ such that

$$
\|w\|_{L^{\infty}\left(0, T ; L^{a}(\Omega)\right)}+\|w\|_{E_{\bar{\kappa}}\left(\Omega_{T}\right)}+\|w\|_{L^{\delta}\left(\Omega_{T}\right)} \leq C\|h\|_{L^{\kappa}\left(\Omega_{T}\right)} .
$$

- If $\kappa=\frac{N+2}{2}$, then there exists $\alpha>0$ depending only on $\Omega$ and $N$ such that $e^{\alpha w} \in$ $L^{\infty}\left(0, T ; L^{2}(\Omega)\right)$ and there exists $C \equiv C(\Omega, N, \kappa)>0$ such that

$$
\left\|e^{\alpha w}\right\|_{L^{\infty}\left(0, T ; L^{2}(\Omega)\right)} \leq C\|h\|_{L^{\kappa}\left(\Omega_{T}\right)} .
$$

- If $\kappa>\frac{N+2}{2}$, then $w \in L^{\infty}\left(\Omega_{T}\right)$ and there exists $C \equiv C(\Omega, N, \kappa)$ such that

$$
\|w\|_{L^{\infty}\left(\Omega_{T}\right)} \leq C\|h\|_{L^{\kappa}\left(\Omega_{T}\right)} .
$$

The case $h \equiv 0$ :

Let $w_{0} \in L^{\tau}(\Omega), \tau \in\left[1, \infty\left[\right.\right.$, then there exist $C_{1}, C_{2}>0$ depending only on $\gamma, \tau, s, N, \Omega$ such that

$$
\|w(., t)\|_{L^{\gamma}(\Omega)} \leq C_{1} \frac{\left\|w_{0}\right\|_{L^{\tau}(\Omega)}}{t^{\frac{N}{2}\left(\frac{1}{\tau}-\frac{1}{\gamma}\right)}}, \quad \tau \leq \gamma \leq+\infty
$$

and

$$
\|\nabla w(., t)\|_{L^{s}(\Omega)} \leq C_{2} \frac{\left\|w_{0}\right\|_{L^{\tau}(\Omega)}}{t^{\frac{N}{2}\left(\frac{1}{\tau}-\frac{1}{s}\right)+\frac{1}{2}}}, \quad \tau \leq s \leq+\infty .
$$

Moreover, $w \in L^{\theta}\left(\Omega_{T}\right)$ for all $\theta<\left(\frac{2}{N}+1\right) \tau$ and $|\nabla w| \in L^{s}\left(\Omega_{T}\right)$ for all $s<\frac{(N+2) \tau}{N+\tau}$. Furthermore, we have

$$
\|w\|_{L^{\theta}\left(\Omega_{T}\right)}+\|w\|_{E_{s}\left(\Omega_{T}\right)} \leq C(\Omega, N, \tau)\left\|w_{0}\right\|_{L^{\tau}(\Omega)} .
$$

Remark 1. The regularity results of Theorem 2.7 and Theorem 2.8 are sharp, in the sense that if the algebraic conditions are violated, one can construct data $h$ or $w_{0}$ such that the above estimates are invalidated and no local solution to the associate equation exists.

Finally, let us recall the famous Schauder fixed-point theorem.

Theorem 2.9 (Schauder fixed-point theorem). Assume that $E$ is a closed convex set of a Banach space $X$. Let $L$ be a continuous and compact mapping from $E$ into itself. Then $L$ has a fixed point in $E$. 


\section{EXISTENCE RESUlts}

As already mentioned in the introduction, in order to simplify the presentation of our results, we will consider separately two cases : the case $(f, g) \neq(0,0)$ with $\left(u_{0}, v_{0}\right) \equiv(0,0)$ and the case $\left(u_{0}, v_{0}\right) \neq(0,0)$ with $(f, g) \equiv(0,0)$.

Henceforth, we denote by $C$ any positive constant that depends only on the data of the problem, and can change from one line to next.

3.1. First case: $\left(u_{0}, v_{0}\right) \neq(0,0)$

The main system is:

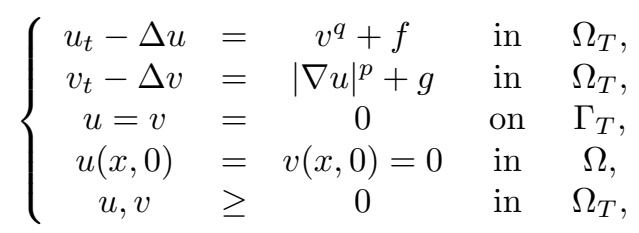

where $\Omega$ is a bounded domain of $\mathbb{R}^{N}$ and $p, q \geq 1$. Here $f$ and $g$ are nonnegative measurable functions with additional assumptions.

The main existence result in this case is the following :

Theorem 3.1. Let $T>0$ and $p, q \geq 1$ with $p q>1$. Assume that $(f, g) \in L^{m}\left(\Omega_{T}\right) \times L^{\sigma}\left(\Omega_{T}\right)$ where $(m, \sigma) \in[1,+\infty)^{2}$ satisfies one of the following conditions:

(3.2) $m, \sigma \in[1, N+2)$ with $p \sigma<\bar{m}=\frac{m(N+2)}{N+2-m}$ and $q m<\frac{(N+1) \bar{\sigma}}{N}=\frac{(N+1)(N+2) \sigma}{N(N+2-\sigma)}$;

(3.3) or, $\sigma \leq N+2 \leq m$, with
a) either $\quad q \leq \frac{\sigma(N+1)}{N(N+2-\sigma)}$
and $p \in[1,+\infty)$,
b) or

$$
\frac{\sigma(N+1)}{N(N+2-\sigma)}<q<\frac{(N+1) \bar{\sigma}}{N} \quad \text { and } \quad p\left[q-\frac{\sigma(N+1)}{N(N+2-\sigma)}\right]<\frac{N+1}{N}
$$

(3.4) or, $m \leq N+2 \leq \sigma$, with

a) either $p \sigma<\bar{m}$ and $q \in[1,+\infty)$,

b) or $\quad p \sigma \geq \bar{m} \quad$ and $\quad q<\frac{(N+1)(N+2)}{N(p[N+2-m]-m)_{+}}$;

(3.5) or, $m, \sigma \geq N+2$, with $q \in[1,+\infty)$ and $p \in[1,+\infty)$.

Then, there exists $T^{*} \leq T$ such that the system (3.1) has a nonnegative solution $(u, v)$. Moreover $(u, v) \in V_{0}^{1, \alpha}\left(\Omega_{T^{*}}\right) \times V_{0}^{1, \beta}\left(\Omega_{T^{*}}\right)$ for all $\alpha<\frac{(N+2) m}{(N+2-m)_{+}}$and $\beta<\frac{(N+2) \sigma}{(N+2-\sigma)_{+}}$.

Remark 2. In order to give some light on the hypotheses (3.2)-(3.5), let us make explicit the size conditions on $(p, q)$ for a given $(m, \sigma)$.

- If $m=\sigma=1$, condition (3.2) holds for all $p<\frac{N+2}{N+1}$ and $q<\frac{N+2}{N}$, which are the maximal 
regularity results of the potential term and gradient term respectively for the solution of parabolic problem with $L^{1}$ data. (See Remark 4).

- If $m=\sigma=2$, condition (3.2) is satisfied for all $p<\frac{N+2}{N}$ and $q<\frac{(N+1)(N+2)}{N^{2}}$.

- If $m>N+2, \sigma=N$, condition (3.3) is satisfied for all $p<\infty$ and for all $q<\frac{N+1}{2}$.

- If $m=N, \sigma=N+2$, condition (3.4) is satisfied for all $p<\frac{N}{2}$ and $q<\infty$.

- If $m=\sigma \geq N+2$, condition (3.5) holds for all $(p, q) \in[1,+\infty)^{2}$.

Remark 3. The above restrictions on the parameters are natural to get existence of solution if we consider elliptic or parabolic systems (or equations) with gradient term. As an example, let us consider a single elliptic equation with gradient term:

$$
-\Delta w=|\nabla w|^{p}+f \text { in } \Omega, w=0 \text { on } \partial \Omega,
$$

with $f \in L^{m}(\Omega)$ and $p>1$. As proved in [38], existence holds under the optimal condition $m>\frac{N}{p^{\prime}}$ which means that $p<\frac{N}{(N-m)_{+}}$. In particular, if $m=2$, the condition $p<\frac{N}{N-2}$ needs to be satisfied. The parabolic case is treated in [27] and [2]. If $p=2$, we can prove that for all $\varepsilon>0$, setting $f(x)=\frac{1}{|x|^{N / 2+\varepsilon}} \notin L^{\frac{N}{2}}\left(B_{1}(0)\right)$, then the corresponding parabolic problem with quadratic gradient term as non local solution.

Proof of Theorem 3.1. We will give the proof under the condition (3.2) i.e

$$
m, \sigma \in(1, N), \quad p \sigma<\frac{m(N+2)}{N+2-m} \text { and } q m<\frac{(N+1)(N+2) \sigma}{N(N+2-\sigma)} .
$$

The other cases follow in a similar way.

The proof will be achieved in several steps.

- First Step : A priori estimates and the construction of the main operator Let $r \geq 1$, recall that $E_{1}\left(\Omega_{T}\right):=L^{1}\left(0, T ; W_{0}^{1,1}(\Omega)\right)$ and $V_{0}^{1, r}\left(\Omega_{T}\right)=L^{\infty}\left(0, T ; L^{1}(\Omega)\right) \cap E_{r}\left(\Omega_{T}\right)$ and define the set

$$
F_{r}\left(\Omega_{T}\right):=\left\{\varphi \in E_{1}\left(\Omega_{T}\right) ; \varphi \in E_{r}\left(\Omega_{T}\right) \cap L^{\infty}\left(0, T ; L^{1}(\Omega)\right) \text { with }\|\varphi\|_{V_{0}^{1, r}\left(\Omega_{T}\right)} \leq M\right\},
$$

where

$$
\|\varphi\|_{V_{0}^{1, r}\left(\Omega_{T}\right)}:=\left(\int_{0}^{T}\|\nabla \varphi\|_{L^{r}(\Omega)}^{r} d t\right)^{\frac{1}{r}}+\|\varphi\|_{L^{\infty}\left(0, T ; L^{1}(\Omega)\right)},
$$

and $M$ is a positive constant that will be chosen later. It is clear that $F_{r}\left(\Omega_{T}\right)$ is a closed convex subset of $E_{1}\left(\Omega_{T}\right)$.

By taking into consideration that $q m<\frac{(N+1) \bar{\sigma}}{N}=\frac{(N+1)(N+2) \sigma}{N(N+2-\sigma)}$, we can choose $r>1$ such that

$$
\frac{q m N}{N+1}<r \leq \bar{\sigma} .
$$


Now, let $\varphi \in F_{r}\left(\Omega_{T}\right)$, according to Proposition 2.1, we have $|\varphi|^{r \frac{N+1}{N}} \in L^{1}\left(\Omega_{T}\right)$. Moreover $q<$ $r \frac{N+1}{N}$, then $|\varphi|^{q} \in L^{1}\left(\Omega_{T}\right)$. Thus we can define $u$ to be the unique weak solution to the problem

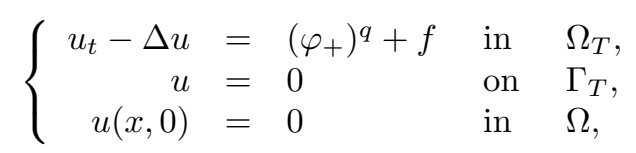

and $u \in V_{0}^{1, s}\left(\Omega_{T}\right)$ for all $s<\frac{N+2}{N+1}$.

Furthermore, (3.6) implies that $q m<r \frac{N+1}{N}$. Therefore $\varphi_{+}^{q} \in L^{m}\left(\Omega_{T}\right)$. Hence applying the estimate (2.7) (Theorem 2.8) and Proposition 2.1 to obtain

$$
\|\nabla u\|_{L^{m}\left(\Omega_{T}\right)} \leq C\left(\|\varphi\|_{L^{q m}\left(\Omega_{T}\right)}^{q}+\|f\|_{L^{m}\left(\Omega_{T}\right)}\right) \leq C\left(\|\varphi\|_{V_{0}^{1, r}\left(\Omega_{T}\right)}^{q}+\|f\|_{L^{m}\left(\Omega_{T}\right)}\right)
$$

with $\bar{m}=\frac{(N+2) m}{N+2-m}$.

In addition, $p \leq p \sigma<\bar{m}$, then $|\nabla u|^{p} \in L^{\sigma}\left(\Omega_{T}\right) \subset L^{1}\left(\Omega_{T}\right)$. Thus we can define $v$ to be the unique weak solution to the problem

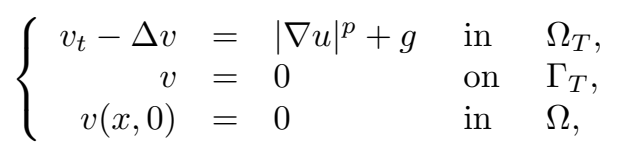

and $v \in V_{0}^{1, s}\left(\Omega_{T}\right)$ for all $s<\frac{N+2}{N+1}$. Applying again the estimate (2.7) (Proposition 2.8) gives

$$
\|\nabla v\|_{L^{\bar{\sigma}}\left(\Omega_{T}\right)} \leq C\left(\|\nabla u\|_{L^{p \sigma}\left(\Omega_{T}\right)}^{p}+\|g\|_{L^{\sigma}\left(\Omega_{T}\right)}\right)
$$

with $\bar{\sigma}=\frac{(N+2) \sigma}{N+2-\sigma}$. Since $p \sigma<\bar{m}=\frac{m(N+2)}{N+2-m}$, by using (3.7), we get

$$
\|\nabla v\|_{L^{\bar{\sigma}}\left(\Omega_{T}\right)} \leq C\left[\|\varphi\|_{L^{q m}\left(\Omega_{T}\right)}^{p q}+\|f\|_{L^{m}\left(\Omega_{T}\right)}^{p}+\|g\|_{L^{\sigma}\left(\Omega_{T}\right)}\right] .
$$

Now, using the fact that $q m \leq r \frac{N+1}{N}$ and $r \leq \bar{\sigma}=\frac{\sigma(N+2)}{N+2-\sigma}$, then according to Proposition 2.1, we have

$$
\|\nabla v\|_{L^{r}\left(\Omega_{T}\right)} \leq C\left[\|\varphi\|_{V_{0}^{1, r}\left(\Omega_{T}\right)}^{p q}+\|f\|_{L^{m}\left(\Omega_{T}\right)}^{p}+\|g\|_{L^{\sigma}\left(\Omega_{T}\right)}\right] .
$$


We estimate now $\|v\|_{L^{\infty}\left(0, T ; L^{1}(\Omega)\right)}$. Using the estimate (2.5) (Theorem 2.7), estimate (3.7) and by Hölder's inequality, we get

$$
\begin{aligned}
\|v\|_{L^{\infty}\left(0, T ; L^{1}(\Omega)\right)} & \leq C\left\||\nabla u|^{p}+g\right\|_{L^{1}\left(\Omega_{T}\right)}=C\left(\|\nabla u\|_{L^{p}\left(\Omega_{T}\right)}^{p}+\|g\|_{L^{1}\left(\Omega_{T}\right)}\right) \\
& \leq C\left(\|\nabla u\|_{L^{m}\left(\Omega_{T}\right)}^{p}+\|g\|_{L^{\sigma}\left(\Omega_{T}\right)}\right) \\
& \leq C\left(\|\varphi\|_{L^{q m}\left(\Omega_{T}\right)}^{p q}+\|g\|_{L^{\sigma}\left(\Omega_{T}\right)}+\|f\|_{L^{m}\left(\Omega_{T}\right)}^{p}\right) \\
& \leq C\left[\|\varphi\|_{V_{0}^{1, r}\left(\Omega_{T}\right)}^{p q}+\|f\|_{L^{m}\left(\Omega_{T}\right)}^{p}+\|g\|_{L^{\sigma}\left(\Omega_{T}\right)}\right] .
\end{aligned}
$$

Thus, combining the above estimates leads to

$$
\|v\|_{V_{0}^{1, r}\left(\Omega_{T}\right)} \leq C\left[\|\varphi\|_{V_{0}^{1, r}\left(\Omega_{T}\right)}^{p q}+\|g\|_{L^{\sigma}\left(\Omega_{T}\right)}+\|f\|_{L^{m}\left(\Omega_{T}\right)}^{p}\right] .
$$

So, the operator

$$
\begin{aligned}
L: F_{r}\left(\Omega_{T}\right) & \longmapsto E_{1}\left(\Omega_{T}\right) \\
\varphi & \longmapsto L(\varphi)=v,
\end{aligned}
$$

is well defined. In addition, if $v$ is a fixed point of $L$ in $F_{r}\left(\Omega_{T}\right)$, then $(u, v)$ solves System (3.1). Thus we just have to show that $L$ has a fixed point in $F_{r}\left(\Omega_{T}\right)$.

- Second step: $L$ is continuous and compact operator.

We begin by proving the continuity of $L$. Let $\left\{\varphi_{n}\right\}_{n} \subset F_{r}\left(\Omega_{T}\right), \varphi \in F_{r}\left(\Omega_{T}\right)$ such that $\varphi_{n} \rightarrow$ $\varphi$ strongly in $E_{1}\left(\Omega_{T}\right)$. Define $v_{n}:=L\left(\varphi_{n}\right)$ and $v:=L(\varphi)$. So $\left(u_{n}, v_{n}\right)$ and $(u, v)$ satisfy

$$
\left\{\begin{array}{ccccc}
\partial_{t} u_{n}-\Delta u_{n} & = & \left(\varphi_{n}^{+}\right)^{q}+f & \text { in } & \Omega_{T}, \\
\partial_{t} u-\Delta u & = & \left(\varphi^{+}\right)^{q}+f & \text { in } & \Omega_{T}, \\
u_{n}=u & = & 0 & \text { on } & \Gamma_{T},
\end{array}\right.
$$

and

$$
\left\{\begin{array}{ccccc}
\partial_{t} v_{n}-\Delta v_{n} & = & \left|\nabla u_{n}\right|^{p}+g & \text { in } & \Omega_{T}, \\
\partial_{t} v-\Delta v & = & |\nabla u|^{p}+g & \text { in } & \Omega_{T}, \\
v_{n}=v & = & 0 & \text { on } & \Gamma_{T} .
\end{array}\right.
$$

Since $\left\{\varphi_{n}\right\}_{n} \subset F_{r}\left(\Omega_{T}\right)$, then

$$
\left\|\varphi_{n}\right\|_{E_{r}\left(\Omega_{T}\right)}+\left\|\varphi_{n}\right\|_{L^{\infty}\left(0, T ; L^{1}(\Omega)\right)} \leq M
$$

Taking into consideration that $\varphi_{n} \rightarrow \varphi$ strongly in $E_{1}\left(\Omega_{T}\right)$, by Proposition 2.1 and by using Vitali's lemma we conclude that $\varphi_{n} \rightarrow \varphi$ strongly in $L^{\gamma}\left(\Omega_{T}\right)$ for all $\gamma<r \frac{N+1}{N}$. Since $q m<r \frac{N+1}{N}$, then $\varphi_{n} \rightarrow \varphi$ strongly in $L^{q m}\left(\Omega_{T}\right)$.

Going back to (3.11) and using estimate (2.7) (Theorem 2.8) to obtain for all $\mu \leq \bar{m}$

$$
\left\|\nabla u_{n}-\nabla u\right\|_{L^{\mu}\left(\Omega_{T}\right)} \leq C\left\|\left(\varphi_{n}^{+}\right)^{q}-\left(\varphi^{+}\right)^{q}\right\|_{L^{m}\left(\Omega_{T}\right)} \rightarrow 0 \text { as } n \rightarrow \infty .
$$

Now, thanks to Theorem $2.7 v_{n} \rightarrow v$ strongly in $E_{s}\left(\Omega_{T}\right)$ for all $s<\frac{N+2}{N+1}$ which implies the continuity of $L$. 
It remains to prove that $L$ is compact. Let $\left\{\varphi_{n}\right\}_{n} \subset F_{r}\left(\Omega_{T}\right)$ be such that $\left\|\varphi_{n}\right\|_{V_{0}^{1, r}\left(\Omega_{T}\right)} \leq C$. Define $v_{n}=L\left(\varphi_{n}\right)$. Since $\left\{\varphi_{n}\right\}_{n} \subset F_{r}\left(\Omega_{T}\right)$, it follows that

$$
\left\|\varphi_{n}\right\|_{E_{r}\left(\Omega_{T}\right)}+\left\|\varphi_{n}\right\|_{L^{\infty}\left(0, T ; L^{1}(\Omega)\right)} \leq M
$$

Therefore, as in the proof of the continuity of $L$, it holds that the sequence $\left\{\varphi_{n}\right\}_{n}$ is bounded in $L^{q m+\varepsilon}\left(\Omega_{T}\right)$ for some $\varepsilon>0$. Define $h_{n}:=\left(\varphi_{n}^{+}\right)^{q}+f$, then $\left\|h_{n}\right\|_{L^{1}\left(\Omega_{T}\right)} \leq C$ for all $n$. Thus, by the compactness result in Theorem 2.7, we obtain that, up to a subsequence, $u_{n} \rightarrow u$ strongly in $E_{s}\left(\Omega_{T}\right)$ for all $s<\frac{N+2}{N+1}$. Taking into consideration that $\left\{u_{n}\right\}_{n}$ is bounded in $E_{\mu}\left(\Omega_{T}\right)$ for all $\mu \leq \bar{m}$ and by Vitali's lemma, we conclude that $v_{n} \rightarrow v$ strongly in $E_{s}\left(\Omega_{T}\right)$ for all $s<\frac{N+2}{N+1}$, in particular for $s=1$. Hence the result follows.

- Third step : To finish our proof, we will choose $M$ and $T^{*}$ such that $L\left(F_{r}\left(\Omega_{T^{*}}\right)\right) \subset F_{r}\left(\Omega_{T^{*}}\right)$. For $s \geq 0$, we consider the concave function

$$
\Upsilon(s):=s^{\frac{1}{p q}}-\widetilde{C} s,
$$

where $\widetilde{C}$ is a universal positive constant (depending only on data) that will specified later.

Using the fact that $p q>1$, then there exists $s_{0}>0$ such that $\Upsilon\left(s_{0}\right)=0, \Upsilon(s)>0$ for all $s \in\left(0, s_{0}\right)$, $\Upsilon(s)<0$ for all $s \in\left(s_{0},+\infty\right)$. Thus, we get the existence of positive constants $\ell$ and $\Lambda^{*}$ such that

$$
\max _{s \geq 0} \Upsilon(s)=\Upsilon(\ell)=\Lambda^{*} .
$$

Moreover

$$
\ell^{\frac{1}{p q}}=\tilde{C}\left(\ell+\frac{\Lambda^{*}}{\tilde{C}}\right) .
$$

Let $\ell>0$ satisfying (3.14), then we can fix $T^{*} \leq T$ such that

$$
\|f\|_{L^{m}\left(\Omega_{T^{*}}\right)}^{p}+\|g\|_{L^{\sigma}\left(\Omega_{T^{*}}\right)} \leq \frac{\Lambda^{*}}{\tilde{C}} .
$$

Setting $M=\ell^{\frac{1}{p q}}$, then thanks to (3.14) and (3.15), we have

$$
\tilde{C}\left(M^{p q}+\|f\|_{L^{m}\left(\Omega_{T^{*}}\right)}^{p}+\|g\|_{L^{\sigma}\left(\Omega_{T^{*}}\right)}\right) \leq M
$$

Now, from (3.10), we deduce that

$$
\|v\|_{V_{0}^{1, r}\left(\Omega_{T^{*}}\right)} \leq C\left[M^{p q}+\|g\|_{L^{\sigma}\left(\Omega_{T^{*}}\right)}+\|f\|_{L^{m}\left(\Omega_{T^{*}}\right)}^{p}\right] \leq M .
$$

Hence $L\left(F_{r}\left(\Omega_{T^{*}}\right)\right) \subset F_{r}\left(\Omega_{T^{*}}\right)$.

Fourth step : Since $L$ is a continuous compact operator with $L\left(F_{r}\left(\Omega_{T^{*}}\right)\right) \subset F_{r}\left(\Omega_{T^{*}}\right)$, using the Schauder fixed-point Theorem we get the existence of $v \in F_{r}\left(\Omega_{T^{*}}\right)$ such that $L(v)=v$. By the maximum principle it holds that $u, v>0$. Thus $(u, v)$ solves system (3.1).

Remark 4. Notice that, if $m=\sigma=1$, assumptions $p<\frac{N+2}{N+1}$ and $q<\frac{N+2}{N}$ are optimal in the sense that if $p \geq \frac{N+2}{N+1}$ or $q \geq \frac{N+2}{N}$, we can show the existence of a suitable data $(f, g) \in L^{1}\left(\Omega_{T}\right) \times L^{1}\left(\Omega_{T}\right)$ such that system (3.1) has no local solution. Assume for example that $p \geq \frac{N+2}{N+1}$ and suppose by 
contradiction that system (3.1) has a local solution for all data $(f, g) \in L^{1}\left(\Omega_{T}\right) \times L^{1}\left(\Omega_{T}\right)$. It is clear that $u$ is a supersolution to the problem

$$
\left\{\begin{array}{ccccc}
w_{t}-\Delta w & = & f(x, t) & \text { in } & \Omega_{T}, \\
w(x, t) & = & 0 & \text { on } \quad \Gamma . \\
w(x, 0) & = & u_{0}(x) & \text { in } & \Omega .
\end{array}\right.
$$

Denoting $w$ the unique local entropy solution to problem (3.17), then $w \leq u$. Let us fix $f \in L^{1}\left(\Omega_{T}\right)$ such that if $\nu_{0}>\frac{N+2}{N}$, then $\iint_{\Omega_{T}} w^{\nu_{0}}(x, t) d x d t=\infty$. (This follows by taking into consideration the optimality of the regularity result in Theorem 2.7, see also $[14,18,44])$.

Now, as $|\nabla u|^{p} \in L^{1}\left(\Omega_{T}\right)$, we deduce from Proposition 2.1 that

$$
\iint_{\Omega_{T}} u^{\nu}(x, t) d x d t \leq C^{\nu}\left(\iint_{\Omega_{T}}|\nabla u(x, t)|^{r} d x d t\right)\left(e s s \sup _{0 \leq t \leq T} \int_{\Omega} u(x, t) d x d t\right)^{\frac{r}{N}}<\infty
$$

with $\nu=p \frac{N+1}{N}$. Since $u \geq w$, then $\iint_{\Omega_{T}} w^{\nu}(x, t) d x d t<\infty$. Hence $\nu \leq \nu_{0}<\frac{N+2}{N}$ and then $p<\frac{N+2}{N+1}$, a contradiction with the hypothesis. Hence we conclude.

In the case where $p=q=1$, we have the next existence result, which improves the existence result obtained in [8] for the elliptic case.

Theorem 3.2. Let $T>0$. Assume that $(f, g) \in L^{1}\left(\Omega_{T}\right) \times L^{1}\left(\Omega_{T}\right)$ with $f, g \supsetneqq 0$ in $\Omega_{T}$. Then, the following system

$$
\left\{\begin{array}{ccccc}
u_{t}-\Delta u & = & v+f & \text { in } & \Omega_{T} \\
v_{t}-\Delta v & = & |\nabla u|+g & \text { in } & \Omega_{T} \\
u=v & = & 0 & \text { on } & \Gamma_{T} \\
u(x, 0) & = & 0 & \text { in } & \Omega, \\
v(x, 0) & = & 0 & \text { in } & \Omega \\
u, v & \geq & 0 & \text { in } & \Omega_{T}
\end{array}\right.
$$

has a solution $(u, v) \in\left(V_{0}^{1, \theta}\left(\Omega_{T}\right)\right)^{2}$ for all $\theta<\frac{N+2}{N+1}$.

Proof. Notice that, in this case we lose the concavity of the real function defined in (3.13). Therefore, we proceed by approximation. By [39], we get the existence of a sequence $\left\{\left(u_{n}, v_{n}\right)\right\}_{n} \subset$ $\left(V_{0}^{1, \theta}\left(\Omega_{T}\right)\right)^{2}$, for all $\theta<\frac{N+2}{N+1}$ such that $\left(u_{n}, v_{n}\right)$ solves the system

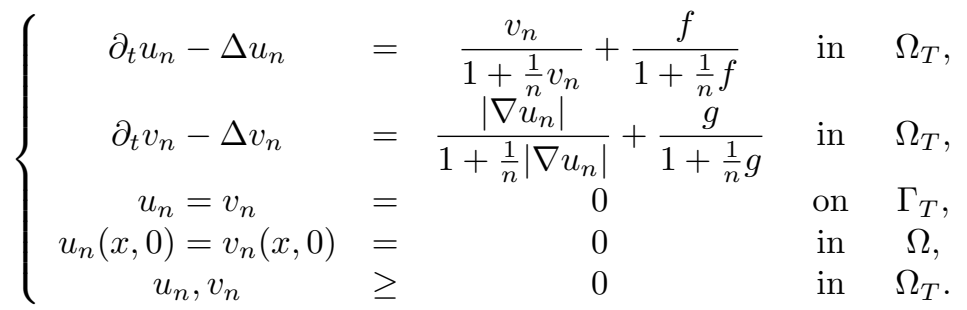

For $s \geq 0$, we consider the function $k(s)=1-\frac{1}{(1+s)^{a}}$ with $a>0$. Let $\widehat{K}(s)=\int_{0}^{s} k(\tau) d \tau$, then $\widehat{K}(s) \geq C_{1} s-C_{2}$ for all $s \geq 0$. 
Using $k\left(v_{n}\right)$ as a test function in the equation of $v_{n}$, it follows that

$$
\int_{\Omega} \widehat{K}\left(v_{n}\right)(x, t) d x+a \int_{0}^{t} \int_{\Omega} \frac{\left|\nabla v_{n}\right|^{2}}{\left(1+v_{n}\right)^{1+a}} d x d \tau \leq \int_{0}^{t} \int_{\Omega}\left|\nabla u_{n}\right| d x d \tau+\int_{0}^{t} \int_{\Omega} g_{n} d x d \tau .
$$

Thanks to Theorem 2.7, we have for all $r<\frac{N+2}{N+1}$ and for all $t \leq T$,

$$
\left(\int_{0}^{t} \int_{\Omega}\left|\nabla u_{n}\right|^{r} d x d \tau\right)^{\frac{1}{r}} \leq C\left(\int_{0}^{t} \int_{\Omega} v_{n} d x d \tau+\int_{0}^{t} \int_{\Omega} f_{n} d x d \tau\right) .
$$

Thus, for $r=1$, we get

$$
\int_{0}^{t} \int_{\Omega}\left|\nabla u_{n}\right| d x d \tau \leq C\left(\int_{0}^{t} \int_{\Omega} v_{n} d x d \tau+\|f\|_{L^{1}\left(\Omega_{T}\right)}\right) .
$$

Going back to (3.20), we obtain

$$
\int_{\Omega} \widehat{K}\left(v_{n}\right)(x, t) d x \leq\left(\int_{0}^{t} \int_{\Omega} v_{n} d x d \tau+\|f\|_{L^{1}\left(\Omega_{T}\right)}+\|g\|_{L^{1}\left(\Omega_{T}\right)}\right) .
$$

Recall that $\widehat{K}\left(v_{n}\right) \geq C_{1} v_{n}-C_{2}$, then by using Gronwall's Lemma we conclude that

$$
\left\|v_{n}\right\|_{L^{1}\left(\Omega_{T}\right)} \leq C \equiv C\left(\Omega_{T},\|f\|_{L^{1}\left(\Omega_{T}\right)},\|g\|_{L^{1}\left(\Omega_{T}\right)}\right) .
$$

Thus, by Theorem 2.7 , we have for all $s_{1}, s_{2}<\frac{N+2}{N+1}$,

$$
\begin{gathered}
\left\|u_{n}\right\|_{\mathcal{C}\left(0, T ; L^{1}(\Omega)\right)}+\left\|u_{n}\right\|_{E_{s_{1}}\left(\Omega_{T}\right)} \leq C, \\
\left\|v_{n}\right\|_{\mathcal{C}\left(0, T ; L^{1}(\Omega)\right)}+\left\|v_{n}\right\|_{E_{s_{2}}\left(\Omega_{T}\right)} \leq C .
\end{gathered}
$$

Hence the existence result follows using the compactness result in Theorem 2.7.

3.2. The case $(f, g)=(0,0)$ and $\left(u_{0}, v_{0}\right) \neq(0,0)$. In this subsection, we suppose that $f \equiv g \equiv 0$ and $\left(u_{0}, v_{0}\right) \in L^{m_{1}}(\Omega) \times L^{\sigma_{1}}(\Omega)$ where $m_{1}, \sigma_{1} \geq 1$. Then, the system (1.1) is reduced to the following one

$$
\left\{\begin{array}{ccccc}
u_{t}-\Delta u & = & v^{q} & \text { in } & \Omega_{T}, \\
v_{t}-\Delta v & =|\nabla u|^{p} & \text { in } & \Omega_{T}, \\
u & = & v=0 & \text { on } & \Gamma_{T}, \\
u(x, 0) & = & u_{0}(x) & \text { in } & \Omega, \\
v(x, 0) & = & v_{0}(x) & \text { in } & \Omega, \\
u, v & \geq & 0 & \text { in } & \Omega_{T} .
\end{array}\right.
$$

The main existence result in this case is the following.

Theorem 3.3. Assume that $\left(u_{0}, v_{0}\right) \in L^{m_{1}}(\Omega) \times L^{\sigma_{1}}(\Omega)$ where $m_{1}, \sigma_{1} \geq 1$. Let $p, q>1$ be such that

$$
\left\{\begin{array}{l}
q<\sigma_{1}\left(\frac{2}{N}+\frac{1}{m_{1}}\right) \\
p<\frac{m_{1}\left(N+\sigma_{1}+1\right)}{\sigma_{1}\left(N+m_{1}\right)} .
\end{array}\right.
$$


Then, for all $T>0$, there exists a positive constant $\mathrm{S}$ depending only on $T$ and the data such that if

$$
\left\|u_{0}\right\|_{L^{m_{1}}(\Omega)}+\left\|v_{0}\right\|_{L^{\sigma_{1}}(\Omega)} \leq \mathrm{S}
$$

System (3.21) has a nonnegative solution $(u, v)$ such that $(u, v) \in V_{0}^{1, \alpha}\left(\Omega_{T}\right) \times V_{0}^{1, \beta}\left(\Omega_{T}\right)$ for all $\alpha<\frac{m_{1}(N+2)}{N+m_{1}}$ and for all $\beta<\frac{\sigma_{1}(N+2)}{N+1}$.

Remark 5. The upper bound for $q$ obtained in the previous Theorem is the same as in the case of nonlinear system with potential nonlinearities. We refer to [32] and [44, Theorem 32.1], for more details about this class of systems.

Proof of Theorem 3.3. In order to prove the main existence result, we will take advantage of the argument used in the proof of Theorem 3.1 obtained in the first part of the paper. Using a suitable change of variable, we will show a relationship between the system (3.21) and the system (3.1) with suitable data $\left(f_{1}, g_{1}\right)$.

Let $\psi$ and $\eta$ to be the unique solutions to the following problems

$$
\left\{\begin{array} { c c c c } 
{ \psi _ { t } - \Delta \psi } & { = } & { 0 } & { \text { in } \Omega _ { T } , } \\
{ \psi ( x , t ) } & { = } & { 0 } & { \text { on } \Gamma _ { T } , } \\
{ \psi ( x , 0 ) } & { = } & { u _ { 0 } ( x ) } & { \text { in } \quad \Omega , }
\end{array} \text { and } \left\{\begin{array}{ccccc}
\eta_{t}-\Delta \eta & = & 0 & \text { in } \Omega_{T}, \\
\eta(x, t) & = & 0 & \text { on } & \Gamma_{T}, \\
\eta(x, 0) & = & v_{0}(x) & \text { in } & \Omega
\end{array}\right.\right.
$$

Thanks to Theorem 2.8, we have

$$
\eta \in L^{\gamma}\left(\Omega_{T}\right) \text { for all } \gamma<\left(\frac{2}{N}+1\right) \sigma_{1} \text { and }|\nabla \psi| \in L^{s}\left(\Omega_{T}\right) \text { for all } s<\frac{(N+2) m_{1}}{N+m_{1}} .
$$

Notice that, if $(u, v)$ solves System (3.21), then $\psi \leq u$ and $\eta \leq v$.

We set $u_{1}=u-\psi$ and $v_{1}=v-\eta$, then $\left(u_{1}, v_{1}\right)$ solves

$$
\left\{\begin{array}{ccccc}
\partial_{t} u_{1}-\Delta u_{1} & = & \left(v_{1}+\eta\right)^{q} & \text { in } & \Omega_{T}, \\
\partial_{t} v_{1}-\Delta v_{1} & = & \left|\nabla u_{1}+\nabla \psi\right|^{p} & \text { in } & \Omega_{T}, \\
u_{1}=v_{1} & = & 0 & \text { on } & \Gamma_{T}, \\
u_{1}(x, 0) & = & 0 & \text { in } & \Omega, \\
v_{1}(x, 0) & = & 0 & \text { in } & \Omega .
\end{array}\right.
$$

Hence in order to show the main existence result, we have just to show that system (3.24) has a non-negative solution.

As above, we fix $1<r<\frac{(N+2) \sigma_{1}}{N+\sigma_{1}}$ very close to $\frac{(N+2) \sigma_{1}}{N+\sigma_{1}}$, then we consider the set

$$
F_{r}\left(\Omega_{T}\right):=\left\{\varphi \in E_{1}\left(\Omega_{T}\right) ; \varphi \in E_{r}\left(\Omega_{T}\right) \cap L^{\infty}\left(0, T ; L^{1}(\Omega)\right) \text { with }\|\varphi\|_{V_{0}^{1, r}\left(\Omega_{T}\right)} \leq \ell^{\frac{1}{p q}}\right\},
$$

where

$$
\|\varphi\|_{V_{0}^{1, r}\left(\Omega_{T}\right)}:=\left(\int_{0}^{T}\|\nabla \varphi\|_{L^{r}(\Omega)}^{r} d t\right)^{\frac{1}{r}}+\|\varphi\|_{L^{\infty}\left(0, T ; L^{1}(\Omega)\right)} .
$$

We define now the operator $L: F_{r}\left(\Omega_{T}\right) \longrightarrow E_{1}\left(\Omega_{T}\right)$ by setting $L(\varphi)=v_{1}$ where $v_{1}$ is the unique solution to problem

$$
\left\{\begin{array}{ccccc}
\partial_{t} v_{1}-\Delta v_{1} & = & \left|\nabla u_{1}+\nabla \psi\right|^{p} & \text { in } & \Omega_{T} \\
v_{1} & = & 0 & \text { on } & \Gamma_{T} \\
v_{1}(x, 0) & = & 0 & \text { in } & \Omega
\end{array}\right.
$$


with $u_{1}$ being the unique solution to the problem

$$
\left\{\begin{aligned}
\partial_{t} u_{1}-\Delta u_{1} & =\left(\eta+\varphi^{+}\right)^{q} & & \text { in } \Omega_{T} \\
u_{1} & =0 & & \text { on } \Gamma_{T} \\
u_{1}(x, 0) & =0 & & \text { in } \Omega
\end{aligned}\right.
$$

It is clear that if $v_{1}$ is a fixed point of $L$ in $F_{r}\left(\Omega_{T}\right)$, then $\left(u_{1}, v_{1}\right)$ solves System (3.24).

Notice that, for a universal constant $C>0$, we have

$$
\left(\eta+\varphi_{+}\right)^{q} \simeq C\left(\varphi_{+}^{q}+\eta^{q}\right)
$$

and

$$
\left|\nabla u_{1}+\nabla \psi\right|^{p} \leq C\left(\left|\nabla u_{1}\right|^{p}+|\nabla \psi|^{p}\right)
$$

We set $f_{1}=C \eta^{q}$ and $g_{1}=C|\nabla \psi|^{p}$. Then thanks to the regularity of $\left(u_{0}, v_{0}\right)$ and by $(3.23)$, we conclude that $f_{1} \in L^{m}\left(\Omega_{T}\right)$ for all $m<\left(\frac{2}{N}+1\right) \frac{\sigma_{1}}{q}$ and $g_{1} \in L^{\sigma}\left(\Omega_{T}\right)$ for all $\sigma<\frac{(N+2) m_{1}}{p\left(N+m_{1}\right)}$.

Since (3.22) holds, then by a direct computations, we verify that $(m, \sigma)$ satisfies condition $(3.2)$. Thus Theorem 3.1 implies that system (3.24) has a solution $\left(u_{1}, v_{1}\right) \in V_{0}^{1, \theta_{1}}(\Omega) \times V_{0}^{1, \theta_{2}}(\Omega)$ for all $\theta_{1}<\frac{m(N+2)}{N+2-m}$ and $\theta_{2}<\frac{(N+2) \sigma}{N+2-\sigma}$.

Recall that $u=u_{1}+\psi$, thus $|\nabla u| \in L^{\theta}\left(\Omega_{T}\right)$ with $\theta=\min \left\{\theta_{1}, \frac{m_{1}(N+2)}{N+m_{1}}\right\}$. Since $m$ can be chosen very close to $\left(\frac{2}{N}+1\right) \frac{\sigma_{1}}{q}$, it holds that

$$
\theta_{1} \simeq \frac{m(N+2)}{N+2-m} \simeq \frac{\sigma_{1}(N+2)}{N q-\sigma_{1}}
$$

which means that for all $\varepsilon>0$, we have

$$
\theta_{1}<\frac{\sigma_{1}(N+2)}{N q-\sigma_{1}}<\theta_{1}+\varepsilon
$$

Now using the fact that $q<\sigma_{1}\left(\frac{2}{N}+\frac{1}{m_{1}}\right)$ to conclude that $\theta_{1}>\frac{m_{1}(N+2)}{N+m_{1}}$. Thus $\theta \simeq \frac{m_{1}(N+2)}{N+m_{1}}$.

In the same way, we obtain that $|\nabla v| \in L^{r}\left(\Omega_{T}\right)$ for all $r \simeq \frac{\sigma_{1}(N+2)}{N+1}$.

To finish, we have just to show, in this case, that the smallness condition (3.15) can be imposed on $\left\|u_{0}\right\|_{L^{m_{1}}(\Omega)}$ and $\left\|v_{0}\right\|_{L^{\sigma_{1}}(\Omega)}$. Recall that from (3.15), we need that

$$
\left\|f_{1}\right\|_{L^{m}\left(\Omega_{T}\right)}^{p}+\left\|g_{1}\right\|_{L^{\sigma}\left(\Omega_{T}\right)} \leq \frac{\Lambda^{*}}{\tilde{C}}
$$

Since $f_{1}=C \eta^{q}$ and $g_{1}=C|\nabla \psi|^{p}$, using estimates (2.10) and (2.11) (Theorem 2.8) yields to

$$
\left\|f_{1}\right\|_{L^{m}\left(\Omega_{T}\right)}^{p}=C\|\eta\|_{L^{q m}\left(\Omega_{T}\right)}^{p q} \leq \tilde{C}\left\|v_{0}\right\|_{L^{\sigma_{1}(\Omega)}}^{p q}\left(\int_{0}^{T} t^{-q m \frac{N}{2}\left(\frac{1}{\sigma_{1}}-\frac{1}{q m}\right)} d t\right)^{\frac{p}{m}}
$$

where $\tilde{C}$ depends only on $p, q, m_{1}, \sigma_{1}$ and $|\Omega|$. Since $q m<\sigma_{1}\left(\frac{2}{N}+1\right)$, then $q m \frac{N}{2}\left(\frac{1}{\sigma_{1}}-\frac{1}{q m}\right)<1$. Thus

$$
\left\|f_{1}\right\|_{L^{m}\left(\Omega_{T}\right)}^{p} \leq \tilde{C}\left\|v_{0}\right\|_{L^{\sigma_{1}(\Omega)}}^{p q} T^{\frac{p}{m}\left(1-q m \frac{N}{2}\left(\frac{1}{\sigma_{1}}-\frac{1}{q m}\right)\right)}
$$


In the same way, we obtain

$$
\left\|g_{1}\right\|_{L^{\sigma}\left(\Omega_{T}\right)}=\tilde{C}\left(\int_{0}^{T} \int_{\Omega}|\nabla \psi|^{p \sigma} d x d t\right)^{\frac{1}{\sigma}} \leq \tilde{C}\left\|u_{0}\right\|_{L^{m_{1}}(\Omega)}^{p} T^{\frac{1}{\sigma}\left(1-p \sigma \frac{N}{2}\left(\frac{1}{m_{1}}-\frac{1}{p \sigma}\right)\right)}
$$

which follows by using the fact that $p \sigma<\frac{(N+2) m_{1}}{N+m_{1}}$. Combining the above estimates, it hods that

$$
\left\|f_{1}\right\|_{L^{m}\left(\Omega_{T}\right)}^{p}+\left\|g_{1}\right\|_{L^{\sigma}\left(\Omega_{T}\right)} \leq \tilde{C}\left(\left\|v_{0}\right\|_{L^{\sigma_{1}}(\Omega)}^{p q} T^{\frac{p}{m}\left(1-q m \frac{N}{2}\left(\frac{1}{\sigma_{1}}-\frac{1}{q m}\right)\right)}+\left\|u_{0}\right\|_{L^{m_{1}}(\Omega)}^{p} T^{\frac{1}{\sigma}\left(1-p \sigma \frac{N}{2}\left(\frac{1}{m_{1}}-\frac{1}{p \sigma}\right)\right)}\right) .
$$

Hence, for $T$ fixed, we can choose $\mathrm{S}>0$ such that if

$$
\left\|u_{0}\right\|_{L^{m_{1}}(\Omega)}+\left\|v_{0}\right\|_{L^{\sigma_{1}(\Omega)}} \leq \mathrm{S}
$$

then

$$
\left\|f_{1}\right\|_{L^{m}\left(\Omega_{T}\right)}^{p}+\left\|g_{1}\right\|_{L^{\sigma}\left(\Omega_{T}\right)} \leq \frac{\Lambda^{*}}{\tilde{C}}
$$

given in (3.25). Thus we conclude.

Remark 6. As in Remark 4, if $m_{1}=\sigma_{1}=1$, we deduce from (3.2) that $p<\frac{N+2}{N+1}$ and $q<\frac{N+2}{N}$, the natural entropy regularity for the potential and the gradient term respectively when dealing with $L^{1}$ data.

3.3. Blow up result. In this subsection we analyze the question of blow-up in the time of the solution to system (3.21) in a suitable norm.

In the potential case, the blow up is based on a suitable convexity argument and Jensen inequality. In our case, and to get a control of the gradient term, we need to use a specifically weighted Hardy-type inequality. More precisely we have the next universal inequality proved in [45, Theorem 1.6].

Theorem 3.4. Assume that $\Omega \subset \mathbb{R}^{N}, N \geq 2$, be a bounded regular domain. Let a $>1$ and suppose that $0<\sigma<a-1$. Setting $d(x):=\operatorname{dist}(x, \partial \Omega)$, then there exists a positive constant $C(\Omega, a, \sigma)$ such that for all $v \in \mathcal{C}_{0}^{\infty}(\Omega)$, we have

$$
\int_{\Omega} d^{\sigma-a}(x)|v(x)|^{a} d x \leq C \int_{\Omega} d^{\sigma}(x)|\nabla v(x)|^{a} d x .
$$

Notice that the above result is not valid in general if $\sigma \geq a-1$. As a consequence we get the next blow-up result.

Theorem 3.5. Let $\lambda_{1}$ the fist eigenvalue of the Laplacian operator with Dirichlet condition and denote $\varphi_{1}$, the associated positive eigenfunction normalized in $L^{1}(\Omega)$. Suppose that $q>1$ and $p>2$, then there exists a positive constant $C(p, q, \Omega)$ such that if

$$
\int_{\Omega}\left(u_{0}^{p}+v_{0}^{q}\right) \varphi_{1} d x>C(p, q, \Omega)
$$

then any solution to the system (3.1) blow up in a finite time in the sense that, for some $T^{*}(p, q, \Omega)<$ $\infty$, we have

$$
\lim _{t \rightarrow T^{*}} \int_{\Omega}\left(u^{p}(x, t)+v^{q}(x, t)\right) \varphi_{1}(x) d x=\infty .
$$


Proof. Using $\varphi_{1}$ as a test function in booth equations of $u, v$, it holds that

$$
\frac{d}{d t} \int_{\Omega} u(x, t) \varphi_{1}(x) d x+\lambda_{1} \int_{\Omega} u(x, t) \varphi_{1}(x) d x \geq \int_{\Omega} v^{q}(x, t) \varphi_{1}(x) d x,
$$

and

$$
\frac{d}{d t} \int_{\Omega} v(x, t) \varphi_{1}(x) d x+\lambda_{1} \int_{\Omega} v(x, t) \varphi_{1}(x) d x \geq \int_{\Omega}|\nabla u(x, t)|^{p} \varphi_{1}(x) d x .
$$

By Jensen inequality we conclude that

$$
\frac{d}{d t} \int_{\Omega} u(x, t) \varphi_{1}(x) d x+\lambda_{1} \int_{\Omega} u(x, t) \varphi_{1}(x) d x \geq\left(\int_{\Omega} v(x, t) \varphi_{1}(x) d x\right)^{q} .
$$

Since $\varphi_{1} \simeq \operatorname{dist}(x, \partial \Omega)$, then

$$
\int_{\Omega}|\nabla u(x, t)|^{p} \varphi_{1}(x) d x \geq C \int_{\Omega}|\nabla u(x, t)|^{p} \operatorname{dist}(x, \partial \Omega) d x .
$$

Recall that $p>2$, using the weighted Hardy inequality in Theorem 3.4 and by Jensen inequality, there results that

$$
\begin{aligned}
\int_{\Omega}|\nabla u(x, t)|^{p} \operatorname{dist}(x, \partial \Omega) d x \geq C(\Omega) & \geq \int_{\Omega} u^{p}(\operatorname{dist}(x, \partial \Omega))^{1-p} d x \geq C \int_{\Omega} u^{p}(x, t) \varphi_{1}(x) d x \\
& \geq C\left(\int_{\Omega} u(x, t) \varphi_{1}(x) d x\right)^{p} .
\end{aligned}
$$

Denoting by $Y(t)=\int_{\Omega} u(x, t) \varphi_{1}(x) d x$ and $Z(t)=\int_{\Omega} v(x, t) \varphi_{1}(x) d x$, it holds that

$$
\left\{\begin{array}{l}
Y^{\prime}(t)+\lambda_{1} Y(t) \geq C Z^{q}(t) \\
Z^{\prime}(t)+\lambda_{1} Z(t) \geq C Y^{p}(t)
\end{array}\right.
$$

Setting $l=\min \{p, q\}>1$, we get

$$
(Y+Z)^{\prime}(t)+\lambda_{1}(Y+Z)(t) \geq Z^{q}(t)+Y^{p}(t) \geq C(Y(t)+Z(t))^{l}-C(Y(t)+Z(t)) .
$$

Denoting by $W(t)=Y(t)+Z(t)$, then

$$
W^{\prime}(t)+C W(t) \geq W^{l}(t) .
$$

Since $l>1$, then we get the existence of positive constant $C_{0}$ such that if $W\left(0>C_{0}\right.$, then there exists $T^{*}>0$, depending only on the data, such that

$$
\lim _{t \rightarrow T^{*}} W(t)=\infty \text {. }
$$

Hence we conclude.

\section{Remark 7.}

(1) The above argument is based on a suitable Hardy (or Poincaré) type inequality. In the case where $p \in(1,2)$, inequality like (3.26) does not holds in general and another approach is needed in order to treat this case. 
(2) Notice that from [1], we know that the associate elliptic system has a solution. Hence we hope that, under suitable conditions of the initial data, existence of a global solution holds. However, the main difficulty is to get a suitable comparison principle (in order to use monotony arguments). In the case of single equation with gradient term, the comparison principle was proved in [3] under natural condition on the exponent of the gradient and following the ideas of [5]. The argument in [3] does not work for system and it seems to be very interesting to find another way in order to prove the associate comparison principle.

\section{Some EXtensions}

Following closely the arguments developed in the previous section, we shall also treat the following nonlinear system, where the gradient terms $|\nabla u|^{p}$ and $|\nabla v|^{q}$ act as a source term.

4.1. First model : Let us consider the system

$$
\left\{\begin{array}{ccccc}
u_{t}-\Delta u & = & |\nabla v|^{q}+f & \text { in } & \Omega_{T}, \\
v_{t}-\Delta v & = & |\nabla u|^{p}+g & \text { in } & \Omega_{T}, \\
u=v & = & 0 & \text { on } & \Gamma_{T} \\
u(x, 0) & = & 0 & \text { in } & \Omega, \\
v(x, 0) & = & 0 & \text { in } & \Omega, \\
u, v & \geq & 0 & \text { in } & \Omega_{T} .
\end{array}\right.
$$

where $(f, g) \in L^{m}\left(\Omega_{T}\right) \times L^{\sigma}\left(\Omega_{T}\right)$.

Notice that the case where $f=g=0$ with non-trivial initial conditions and $p, q \leq 2$ was treated in [6]. In that paper, the authors showed the existence of global solutions of the associated Cauchy problem under additional assumptions on $p, q$ and the initial data.

Without the condition $p, q \leq 2$, and under some natural conditions on $f$ and $g$, we are able to prove the next existence result.

Theorem 4.1. Suppose that $m, \sigma \in[1, N+2)$ and let $p, q$ be such that

$$
\left\{\begin{array}{c}
p \sigma<\bar{m}=\frac{m(N+2)}{N+2-m} \\
q m<\bar{\sigma}=\frac{\sigma(N+2)}{N+2-\sigma} .
\end{array}\right.
$$

Then there exists $T^{*} \leq T$ such that system (4.1) has a nonnegative solution $(u, v) \in V_{0}^{1, \alpha}\left(\Omega_{T^{*}}\right) \times$ $V_{0}^{1, \beta}\left(\Omega_{T^{*}}\right)$ for all $\alpha<\bar{m}$ and $\beta<\bar{\sigma}$.

Proof. The proof follows closely the arguments of Section 3, and uses the same fixed-point arguments. However, for the reader's convenience we include here some details.

According to (4.2), let us fix $r>1$ such that $\frac{q m N}{N+1}<r<\bar{\sigma}$ and define the set

$$
F_{r}\left(\Omega_{T}\right):=\left\{\varphi \in E_{1}\left(\Omega_{T}\right) ; \varphi \in E_{r}\left(\Omega_{T}\right) \cap L^{\infty}\left(0, T ; L^{1}(\Omega)\right) \text { with }\|\varphi\|_{V_{0}^{1, r}\left(\Omega_{T}\right)} \leq \ell^{\frac{1}{p q}}\right\}
$$

Then $F_{r}\left(\Omega_{T}\right)$ is a closed convex subset of $E_{1}\left(\Omega_{T}\right)$. 
Now, define the operator

$$
\begin{aligned}
L: F_{r}\left(\Omega_{T}\right) & \longmapsto E_{1}\left(\Omega_{T}\right) \\
\varphi & \longmapsto L(\varphi)=v
\end{aligned}
$$

where $v$ is the unique solution to problem

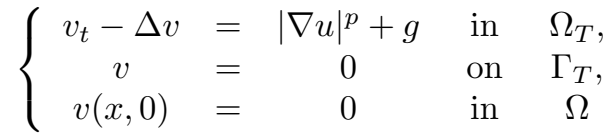

with $u$ being the unique solution of the problem

$$
\left\{\begin{array}{ccccc}
u_{t}-\Delta u & = & |\nabla \varphi|^{q}+f & \text { in } & \Omega_{T}, \\
u & = & 0 & \text { on } & \Gamma_{T}, \\
u(x, 0) & = & 0 & \text { in } & \Omega .
\end{array}\right.
$$

It is clear that if $v$ is a fixed point of $L$ in $F_{r}\left(\Omega_{T}\right)$, then $(u, v)$ solves the system (4.1).

Since $q m<r$, then $|\nabla \varphi|^{q} \in L^{1}\left(\Omega_{T}\right)$. Thus $u$ is well defined and $u \in V_{0}^{1, \alpha}\left(\Omega_{T}\right)$ for all $\alpha<\frac{N+2}{N+1}$.

By Theorem 2.8, we get

$$
\|\nabla u\|_{L^{\bar{m}}\left(\Omega_{T}\right)} \leq C\left\||\nabla \varphi|^{q}+f\right\|_{L^{m}\left(\Omega_{T}\right)} \leq C\left(\|\nabla \varphi\|_{L^{q m}\left(\Omega_{T}\right)}^{q}+\|f\|_{L^{m}\left(\Omega_{T}\right)}\right)
$$

Now, since $p \leq \bar{m}$ then $|\nabla u|^{p}+g \in L^{1}\left(\Omega_{T}\right)$. Thus $v$ is well defined and, at least $v \in V_{0}^{1, \alpha}\left(\Omega_{T}\right)$ for all $\alpha<\frac{N+2}{N+1}$. Thus $L$ is well defined. In the same way, it holds that

$$
\|\nabla v\|_{L^{\bar{\sigma}}\left(\Omega_{T}\right)} \leq C\left[\|\nabla u\|_{L^{p \sigma}\left(\Omega_{T}\right)}^{p}+\|g\|_{L^{\sigma}\left(\Omega_{T}\right)}\right],
$$

with $\bar{\sigma}=\frac{\sigma(N+2)}{N+2-\sigma}$. Since $p \sigma<\bar{m}$, and using inequality (4.3), we obtain

$$
\|\nabla v\|_{L^{\bar{\sigma}}\left(\Omega_{T}\right)} \leq C\left[\|\nabla \varphi\|_{L^{q m}\left(\Omega_{T}\right)}^{p q}+\|f\|_{L^{m}\left(\Omega_{T}\right)}^{p}+\|g\|_{L^{\sigma}\left(\Omega_{T}\right)}\right] .
$$

Since $q m<r<\bar{\sigma}$, by using Hölder's inequality and Proposition 2.1 we obtain

$$
\|\nabla v\|_{L^{r}\left(\Omega_{T}\right)} \leq C\left[\|\varphi\|_{V_{0}^{1, r}\left(\Omega_{T}\right)}^{p q}+\|f\|_{L^{m}\left(\Omega_{T}\right)}^{p}+\|g\|_{L^{\sigma}\left(\Omega_{T}\right)}\right] .
$$

Now, by Theorem 2.7 , we reach that

$$
\|v\|_{L^{\infty}\left(0, T ; L^{1}(\Omega)\right)} \leq C\left[\|\varphi\|_{V_{0}^{1, r}\left(\Omega_{T}\right)}^{p q}+\|g\|_{L^{\sigma}\left(\Omega_{T}\right)}+\|f\|_{L^{m}\left(\Omega_{T}\right)}^{p}\right] .
$$

Thus

$$
\|v\|_{V_{0}^{1, r}\left(\Omega_{T}\right)} \leq C\left[\|\varphi\|_{V_{0}^{1, r}\left(\Omega_{T}\right)}^{p q}+\|f\|_{L^{m}\left(\Omega_{T}\right)}^{p}+\|g\|_{L^{\sigma}\left(\Omega_{T}\right)}\right] .
$$

It is clear that the above estimate holds in $\Omega_{T^{*}}$ for all $T^{*} \leq T$. Hence, by choosing $T^{*}<T$ such that

we have $L\left(F_{r}\left(\Omega_{T^{*}}\right)\right) \subset F_{r}\left(\Omega_{T^{*}}\right)$.

$$
C\left[\ell+\|f\|_{L^{m}\left(\Omega_{T^{*}}\right)}^{p}+\|g\|_{L^{\sigma}\left(\Omega_{T^{*}}\right)}\right] \leq \ell^{\frac{1}{p q}},
$$

Now the continuity and the compactness property of $L$ follow as in the proof of Theorem 3.1. Hence we conclude. 
4.2. Second model : We deal now with the system

$$
\left\{\begin{array}{ccccc}
u_{t}-\Delta u & =|\nabla v|^{q} & \text { in } & \Omega_{T}, \\
v_{t}-\Delta v & =|\nabla u|^{p} & \text { in } & \Omega_{T}, \\
u & = & v=0 & \text { on } & \Gamma_{T} \\
u(x, 0) & = & u_{0}(x) & \text { in } & \Omega \\
v(x, 0) & = & v_{0}(x) & \text { in } & \Omega \\
u, v & \geq & 0 & \text { in } & \Omega_{T} .
\end{array}\right.
$$

where $\left(u_{0}, v_{0}\right) \in L^{m_{1}}\left(\Omega_{T}\right) \times L^{\sigma_{1}}\left(\Omega_{T}\right)$. We have the next existence result.

Theorem 4.2. Assume that $\left(u_{0}, v_{0}\right) \in L^{m_{1}}(\Omega) \times L^{\sigma_{1}}(\Omega)$ where $m_{1}, \sigma_{1} \geq 1$. Let $p, q>1$ be such that

$$
\left\{\begin{aligned}
q & <\frac{\sigma_{1}\left(N+2 m_{1}\right)}{m_{1}\left(N+\sigma_{1}\right)} \\
p & <\frac{m_{1}\left(N+2 \sigma_{1}\right)}{\sigma_{1}\left(N+m_{1}\right)}
\end{aligned}\right.
$$

Then, for all $T>0$, there exists a positive constant $\mathrm{S}$ depending on $T$ and the data such that if

$$
\left\|u_{0}\right\|_{L^{m_{1}}(\Omega)}+\left\|v_{0}\right\|_{L^{\sigma_{1}(\Omega)}} \leq \mathrm{S}
$$

System (4.5) has a nonnegative solution $(u, v)$ such that $(u, v) \in V_{0}^{1, \alpha}\left(\Omega_{T}\right) \times V_{0}^{1, \beta}\left(\Omega_{T}\right)$ for all $\alpha<\frac{m_{1}(N+2)}{N+m_{1}}$ and for all $\beta<\frac{\sigma_{1}(N+2)}{N+\sigma_{1}}$.

Proof. We follow closely the change of variables used in the proof of Theorem 3.3. Let $\psi$ and $\eta$ to be the unique solutions to the following problems

$$
\left\{\begin{array} { c c c c c } 
{ \psi _ { t } - \Delta \psi } & { = } & { 0 } & { \text { in } \quad \Omega _ { T } , } \\
{ \psi ( x , t ) } & { = } & { 0 } & { \text { on } \quad \Gamma _ { T } , } \\
{ \psi ( x , 0 ) } & { = } & { u _ { 0 } ( x ) } & { \text { in } \quad \Omega , }
\end{array} \quad \text { and } \left\{\begin{array}{ccccc}
\eta_{t}-\Delta \eta & = & 0 & \text { in } & \Omega_{T} \\
\eta(x, t) & = & 0 & \text { on } & \Gamma_{T} \\
\eta(x, 0) & = & v_{0}(x) & \text { in } & \Omega
\end{array}\right.\right.
$$

Thanks to Theorem 2.8, we have

$$
|\nabla \eta| \in L^{\gamma}\left(\Omega_{T}\right) \text { for all } \gamma<\frac{(N+2) \sigma_{1}}{N+\sigma_{1}} \text { and }|\nabla \psi| \in L^{s}\left(\Omega_{T}\right) \text { for all } s<\frac{(N+2) m_{1}}{N+m_{1}} \text {. }
$$

We set $u_{1}=u-\psi$ and $v_{1}=v-\eta$, then $\left(u_{1}, v_{1}\right)$ solves

$$
\left\{\begin{array}{ccccc}
\partial_{t} u_{1}-\Delta u_{1} & = & \left|\nabla v_{1}+\nabla \eta\right|^{q} & \text { in } & \Omega_{T}, \\
\partial_{t} v_{1}-\Delta v_{1} & = & \left|\nabla u_{1}+\nabla \psi\right|^{p} & \text { in } & \Omega_{T}, \\
u_{1}=v_{1} & = & 0 & \text { on } & \Gamma_{T}, \\
u_{1}(x, 0) & = & 0 & \text { in } & \Omega, \\
v_{1}(x, 0) & = & 0 & \text { in } & \Omega .
\end{array}\right.
$$

Hence in order to show the main existence result, we have just to show that system (4.8) has a non-negative solution.

For $r$ close to $\frac{(N+2) \sigma_{1}}{N+\sigma_{1}}$, we consider the set

$$
F_{r}\left(\Omega_{T}\right):=\left\{\varphi \in E_{1}\left(\Omega_{T}\right) ; \varphi \in E_{r}\left(\Omega_{T}\right) \cap L^{\infty}\left(0, T ; L^{1}(\Omega)\right) \text { with }\|\varphi\|_{V_{0}^{1, r}\left(\Omega_{T}\right)} \leq \ell^{\frac{1}{p q}}\right\} .
$$


We define now the operator $L: F_{r}\left(\Omega_{T}\right) \longrightarrow E_{1}\left(\Omega_{T}\right)$ by setting $L(\varphi)=v_{1}$ where $v_{1}$ is the unique solution to problem

$$
\left\{\begin{array}{ccccc}
\partial_{t} v_{1}-\Delta v_{1} & = & \left|\nabla u_{1}+\nabla \psi\right|^{p} & \text { in } & \Omega_{T}, \\
v_{1} & = & 0 & \text { on } & \Gamma_{T}, \\
v_{1}(x, 0) & = & 0 & \text { in } & \Omega,
\end{array}\right.
$$

with $u_{1}$ being the unique solution to the problem

$$
\left\{\begin{aligned}
\partial_{t} u_{1}-\Delta u_{1} & =|\nabla \eta+\nabla \varphi|^{q} & & \text { in } \Omega_{T}, \\
u_{1} & =0 & & \text { on } \Gamma_{T}, \\
u_{1}(x, 0) & =0 & & \text { in } \Omega .
\end{aligned}\right.
$$

It is clear that if $v_{1}$ is a fixed point of $L$ in $F_{r}\left(\Omega_{T}\right)$, then $\left(u_{1}, v_{1}\right)$ solves the system (4.8).

Following the same approach as in the proof of Theorem 3.3 we reach to the desired result.

4.3. Third model : Finally let us analyze the following system

$$
\left\{\begin{array}{ccccc}
u_{t}-\Delta u & = & u|\nabla v|^{q}+f & \text { in } & \Omega_{T}, \\
v_{t}-\Delta v & = & v|\nabla u|^{p}+g & \text { in } & \Omega_{T}, \\
u & = & v=0 & \text { on } & \Gamma_{T}, \\
u(x, 0) & = & 0 & \text { in } & \Omega, \\
v(x, 0) & = & 0 & \text { in } & \Omega_{T}, \\
u, v & \geq & 0 & \text { in } & \Omega_{T}, .
\end{array}\right.
$$

which is more involved due to the presence of an interaction term in both equations (the dependence on $u$ and $v$ appears in the two equations).

Notice that, in the case of one equation and in the particular case $p=2$, the problem

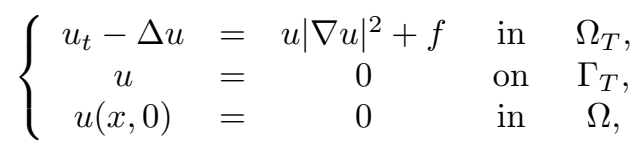

was studied deeply in [27]. The authors proved the existence of a solution to (4.10) if $f \in L^{m}\left(\Omega_{T}\right)$ with $m>\frac{N+2}{2}$. See also [26] for some extensions.

In the sprit of the results obtained in [27] and [26] and keeping free the values of $p, q>1$, we have the next existence result for System (4.9).

Theorem 4.3. Suppose that $(f, g) \in L^{m}\left(\Omega_{T}\right) \times L^{\sigma}\left(\Omega_{T}\right)$ with $1<m, \sigma<N+2$. Let $p, q>1$ be such that

$$
\left\{\begin{aligned}
p \sigma & <\frac{m(N+2+N \sigma)}{(N+2-m)(N+1)}, \\
q m & <\frac{\sigma(N+2+N m)}{(N+2-\sigma)(N+1)} .
\end{aligned}\right.
$$

Then System (4.9) has a nonnegative solution $(u, v) \in V_{0}^{1, \alpha}\left(\Omega_{T}\right) \times V_{0}^{1, \beta}\left(\Omega_{T}\right)$ for all $\alpha<\frac{(N+2) m}{(N+2-m)_{+}}$ and $\beta<\frac{(N+2) \sigma}{(N+2-\sigma)_{+}}$. 
Proof. We follow closely the argument used in the proof of Theorem 4.1. However, taking into consideration the structure of System (4.9), some technical modifications are needed.

Define the function

$$
\Upsilon_{1}(s):=s-\widetilde{C}\left(s^{1+q}+s^{1+p}\right),
$$

with $\widetilde{C}$ being a universal positive constant depending only on the data. Since $q, p>1$, then we get the existence of a unique value $\ell$ such that

$$
\max _{s \geq 0} \Upsilon_{1}(s)=\Upsilon_{1}(\ell)=\Lambda^{*}
$$

Thus

$$
\ell=\tilde{C}\left(\ell^{1+q}+\ell^{1+p}+\frac{\Lambda^{*}}{\tilde{C}}\right) .
$$

By a continuity argument, we choose $0<T^{*}<T$ such that

$$
\|f\|_{L^{m}\left(\Omega_{T^{*}}\right)}+\|g\|_{L^{\sigma}\left(\Omega_{T^{*}}\right)} \leq \frac{\Lambda^{*}}{\tilde{C}},
$$

hence

$$
\tilde{C}\left(\ell^{1+q}+\ell^{1+p}+\|f\|_{L^{m}\left(\Omega_{T^{*}}\right)}+\|g\|_{L^{\sigma}\left(\Omega_{T^{*}}\right)}\right) \leq \ell
$$

Now, we define the set

$$
\begin{aligned}
& F_{r, \theta}\left(\Omega_{T^{*}}\right)=\left\{(\varphi, \psi) \in E_{1}\left(\Omega_{T^{*}}\right) \times E_{1}\left(\Omega_{T^{*}}\right),\right. \\
& \left.(\varphi, \psi) \in V_{0}^{1, r}\left(\Omega_{T^{*}}\right) \times V_{0}^{1, \theta}\left(\Omega_{T^{*}}\right) \text { and }\|\varphi\|_{V_{0}^{1, r}\left(\Omega_{T^{*}}\right)}+\|\psi\|_{V_{0}^{1, \theta}\left(\Omega_{T^{*}}\right)} \leq \ell\right\},
\end{aligned}
$$

where $r$ and $\theta$ are chosen such that

$$
\left\{\begin{array}{l}
\frac{p \sigma(N+1)}{\theta(N+1)-N \sigma}<r<\frac{m(N+2)}{N+2-m}:=\bar{m}, \\
\frac{q m(N+1)}{r(N+1)-N m}<\theta<\frac{\sigma(N+2)}{N+2-\sigma}:=\bar{\sigma} .
\end{array}\right.
$$

Notice that, under the condition (4.11), we get the existence of $(r, \theta)$ closed to $(\bar{m}, \bar{\sigma})$ such that the condition (4.14) holds.

It is clear that $F_{r, \theta}\left(\Omega_{T^{*}}\right)$ is a closed convex subset of $E_{1}\left(\Omega_{T^{*}}\right) \times E_{1}\left(\Omega_{T^{*}}\right)$.

Now consider the operator

$$
\begin{aligned}
& L: F_{r, \theta}\left(\Omega_{T^{*}}\right) \longmapsto E_{1}\left(\Omega_{T^{*}}\right) \times E_{1}\left(\Omega_{T^{*}}\right) \\
& \bar{\varphi}=(\varphi, \psi) \quad \longmapsto \quad L(\varphi, \psi)=(u, v)
\end{aligned}
$$

where $u$ and $v$ solve respectively the following problems

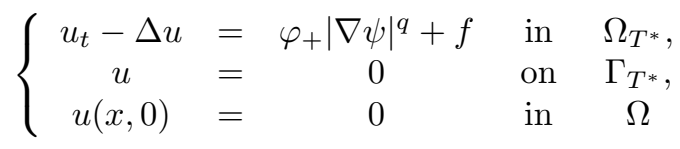


and

$$
\left\{\begin{array}{ccccc}
v_{t}-\Delta v & = & \psi_{+}|\nabla \varphi|^{p}+g & \text { in } & \Omega_{T^{*}} \\
v & = & 0 & \text { on } & \Gamma_{T^{*}} \\
v(x, 0) & = & 0 & \text { in } & \Omega
\end{array}\right.
$$

Of course, if $(u, v)$ is a fixed point of $L$ in $F_{r, \theta}\left(\Omega_{T^{*}}\right)$, then $(u, v)$ solves System (4.9).

We claim that if $(\varphi, \psi) \in F_{r}\left(\Omega_{T^{*}}\right)$, then

$$
\left\|\varphi_{+}|\nabla \psi|^{q}\right\|_{L^{m}\left(\Omega_{T^{*}}\right)} \leq C\|\varphi\|_{V_{0}^{1, r}\left(\Omega_{T^{*}}\right)} \times\|\psi\|_{V_{0}^{1, r}\left(\Omega_{T^{*}}\right)}^{q} .
$$

Indeed, we have

$$
\left\|\varphi_{+}|\nabla \psi|^{q}\right\|_{L^{m}\left(\Omega_{T^{*}}\right)}^{m}=\iint_{\Omega_{T^{*}}} \varphi_{+}^{m}|\nabla \psi|^{q m} d x d t \leq \iint_{\Omega_{T^{*}}}|\varphi|^{m}|\nabla \psi|^{q m} d x d t
$$

Hence using Hölder's inequality gives

$$
\left\|\varphi_{+}|\nabla \psi|^{q}\right\|_{L^{m}\left(\Omega_{T^{*}}\right)}^{m} \leq C\left(\iint_{\Omega_{T^{*}}}|\varphi|^{\nu} d x d t\right)^{\frac{m}{\nu}}\left(\iint_{\Omega_{T^{*}}}|\nabla \psi|^{q m \frac{\nu}{\nu-m}} d x d t\right)^{\frac{\nu-m}{\nu}},
$$

where $\nu=r \frac{N+1}{N}$. Moreover $q m \frac{\nu}{\nu-m}=q m \frac{r(N+1)}{r(N+1)-m N}$. Thus, by the definition of $r$ and $\theta$, it holds that $q m \frac{\nu}{\nu-m}<\theta$.

Going back to (4.15) and applying Proposition 2.1 to obtain

$$
\left\|\varphi_{+}|\nabla \psi|^{q}\right\|_{L^{m}\left(\Omega_{\left.T^{*}\right)}\right.}^{m} \leq C\left(\iint_{\Omega_{T^{*}}}|\varphi|^{\nu} d x d t\right)^{\frac{m}{\nu}}\left(\iint_{\Omega_{T^{*}}}|\nabla \psi|^{\theta} d x d t\right)^{\frac{q m}{\theta}} \leq C\|\varphi\|_{V_{0}^{1, r}\left(\Omega_{T^{*}}\right)}^{m} \times\|\psi\|_{V_{0}^{1, \theta}\left(\Omega_{T^{*}}\right)}^{q m},
$$

and hence the claim follows.

In a symmetric way and since $p \sigma \frac{\theta(N+1)}{\theta(N+1)-\sigma N}<r$, we obtain that

$$
\left\|\psi_{+}|\nabla \varphi|^{p}\right\|_{L^{\sigma}\left(\Omega_{T^{*}}\right)} \leq C\|\psi\|_{V_{0}^{1, \theta}\left(\Omega_{T^{*}}\right)} \times\|\varphi\|_{V_{0}^{1, r}\left(\Omega_{T^{*}}\right)}^{p}
$$

Thus $u, v$ are well defined and $(u, v) \in E_{1}\left(\Omega_{T^{*}}\right) \times E_{1}\left(\Omega_{T^{*}}\right)$. Now, by Theorem 2.8, we get

$$
\begin{gathered}
\|\nabla u\|_{L^{\bar{m}}\left(\Omega_{T^{*}}\right)} \leq C\left(\Omega_{T^{*}}\right)\left(\|\varphi\|_{V_{0}^{1, r}\left(\Omega_{T^{*}}\right)} \times\|\psi\|_{V_{0}^{1, \theta}\left(\Omega_{T^{*}}\right)}^{q}+\|f\|_{L^{m}\left(\Omega_{T^{*}}\right)}\right), \\
\|\nabla v\|_{L^{\bar{\sigma}}\left(\Omega_{T^{*}}\right)} \leq C\left(\Omega_{T^{*}}\right)\left(\|\psi\|_{V_{0}^{1, \theta}\left(\Omega_{T^{*}}\right)} \times\|\varphi\|_{V_{0}^{1, r}\left(\Omega_{T^{*}}\right)}^{p}+\|g\|_{L^{\sigma}\left(\Omega_{T^{*}}\right)}\right),
\end{gathered}
$$


where $\bar{m}=\frac{m(N+2)}{N+2-m}$ and $\bar{\sigma}=\frac{\sigma(N+2)}{N+2-\sigma}$. Since $r<\bar{m}$ and $\theta<\bar{\sigma}$, then $(u, v) \in V_{0}^{1, r}\left(\Omega_{T^{*}}\right) \times$ $V_{0}^{1, \theta}\left(\Omega_{T^{*}}\right)$ and

$$
\begin{aligned}
\|u\|_{V_{0}^{1, r}\left(\Omega_{T^{*}}\right)}+\|v\|_{V_{0}^{1, r}\left(\Omega_{T^{*}}\right)} & \leq C\left(\Omega_{T^{*}}\right)\left(\|\varphi\|_{V_{0}^{1, r}\left(\Omega_{T^{*}}\right)}\|\psi\|_{V_{0}^{1, \theta}\left(\Omega_{T^{*}}\right)}^{q}\right. \\
& \left.+\|\psi\|_{V_{0}^{1, \theta}\left(\Omega_{T^{*}}\right)}\|\varphi\|_{V_{0}^{1, r}\left(\Omega_{T^{*}}\right)}^{p}+\|f\|_{L^{m}\left(\Omega_{T^{*}}\right)}+\|g\|_{L^{\sigma}\left(\Omega_{T^{*}}\right)}\right) \\
& \leq C\left(\Omega_{T^{*}}\right)\left(\ell^{1+q}+\ell^{1+p}+\|f\|_{L^{m}\left(\Omega_{T^{*}}\right)}+\|g\|_{L^{\sigma}\left(\Omega_{T^{*}}\right)}\right) \\
& \leq \ell .
\end{aligned}
$$

The last estimate follows by (4.13). Therefore we conclude that $L\left(F_{r, \theta}\left(\Omega_{T^{*}}\right)\right) \subset F_{r, \theta}\left(\Omega_{T^{*}}\right)$.

The rest of the proof is the same as the proof of Theorem 4.1.

Remark 8. To illustrate our previous result, let us give some examples of $p, q, m$ and $\sigma$.

- If $m, \sigma>N+2$, the existence result holds for all $p, q>1$.

- If $p=q=1$ and $m=\sigma$, a sufficient condition on $m$ that guarantees the existence of a solution is $m=\sigma>\frac{N(N+2)}{2 N+1}$.

- If $p=q=2$ and $m=\sigma$, condition (4.11) implies that $m=\sigma>\frac{(N+2)(2 N+1)}{3 N+2}$.

- If $m=\sigma=2$, then the condition (4.11) implies that

$$
\left\{\begin{array}{c}
p<\frac{3 N+2}{N(N+1)} \\
q<\frac{3 N+2}{N(N+1)}
\end{array}\right.
$$

Or $\frac{3 N+2}{N(N+1)}>1$ if $N \leq 2$. Hence for $N \geq 3$, it seems that the condition $p, q<1$ is necessary as it will be shown in the next proposition.

Proposition 4.4. Assume that $m=\sigma=2$. Then for all $p, q>1$, there exist $f, g \in L^{2}\left(\Omega_{T}\right)$ such that System (4.9) has no solution at least for $N$ large.

Proof. We consider the case where $0 \in \Omega$ and $f(x, t)=g(x, t)=\frac{1}{|x|^{\alpha}}$ where $2<\alpha<\frac{N}{2}$. If $(u, v)$ is a solution to System (4.9), then using a suitable comparison principle it holds that :

$$
u(x, t), v(x, t) \geq \frac{C}{|x|^{\alpha-2}} \text { in } B_{r}(0) \times\left(t_{1}, t_{2}\right) \subset \subset \Omega_{T} .
$$

Thus

$$
\iint_{\Omega_{T}}|x|^{-(\alpha-2)}|\nabla u|^{p} d x d t+\iint_{\Omega_{T}}|x|^{-(\alpha-2)}|\nabla v|^{q} d x d t<\infty
$$


Hence applying Caffarelli-Kohn-Nirenberg's inequality (see [22]) to obtain

$$
\int_{0}^{T}\left(\int_{\Omega} \frac{u^{p^{*}}}{|x|^{\frac{p^{*}}{p}(\alpha-2)}} d x\right)^{\frac{p}{p^{*}}} d t+\int_{0}^{T}\left(\int_{\Omega} \frac{v^{q^{*}}}{|x|^{\frac{q^{*}}{q}(\alpha-2)}} d x\right)^{\frac{q}{q^{*}}} d t<\infty .
$$

Now, using the behavior of $u, v$ at the origin, it holds that

$$
\int_{B_{r}(0)} \frac{1}{|x|^{p^{*}(\alpha-2)+\frac{p^{*}}{p}(\alpha-2)}} d x+\int_{B_{r}(0)} \frac{1}{|x|^{q^{*}(\alpha-2)+\frac{q^{*}}{q}(\alpha-2)}} d x \leq C(T, \Omega) .
$$

Thus

$$
p^{*}(\alpha-2)+\frac{p^{*}}{p}(\alpha-2)<N \text { and } q^{*}(\alpha-2)+\frac{q^{*}}{q}(\alpha-2)<N .
$$

Let $p, q>1$ fixed, by choosing $N>\max \left\{\frac{2 p+4}{p-1}, \frac{2 q+4}{q-1}\right\}$, we get the existence of $\alpha<\frac{N}{2}$ such that

$$
p^{*}(\alpha-2)+\frac{p^{*}}{p}(\alpha-2) \geq N \text { and } q^{*}(\alpha-2)+\frac{q^{*}}{q}(\alpha-2) \geq N,
$$

which is a contradiction with the existence result.

Remark 9. In a forthcoming work we will treat the case of the Cauchy problem, and analyze questions such global existence versus finite-time blow-up. Notice that, for a semi-linear reactiondiffusion system with potential terms, the existence of a Fujita-type exponent has been proved in [32], see also [44, Theorem 32.7] for an alternative proof in the subcritical case.

\section{Acknowledgments:}

The authors would like to express their gratitude to the anonymous referee for his/her comments and suggestions that improve the last version of the manuscript.

Part of this work was done while the first three authors were visiting l'Institut Elie Cartan, Université de Lorraine. They would like to thank the Institute for the warm hospitality.

\section{REFERENCES}

[1] B. Abdellaoui, A. Attar, E.-H. Laamri, On the existence of positive solutions to semilinear elliptic systems involving gradient term. .J. Appl. Anal. 98 (2019), no. 7, 1289-1306.

[2] B. Abdellaoui, A. Dall'Aglio, I. Peral, Regularity and nonuniqueness results for parabolic problems arising in some physical models, having natural growth in the gradient. J. Math. Pures Appl. 90 (2008), 242-269.

[3] B. Abdellaoui, Y. Nasri, A. Primo, Strong comparison results for quasilinear elliptic equation with gradient term. Mediterr. J. Math. 10 (2013), no. 1, 289-311.

[4] N. Alaa, Solutions faibles d'équations paraboliques quasilinéaires avec données initiales mesures. J. Ann. Math. Blaise Pascal, 3 (1996), no. 2, 1-15.

[5] N. Alaa, M. Pierre, Weak solutions of some quasilinear elliptic equations with data measures. SIAM J. Math. Anal. 24 (1993), no. 1, 23-35.

[6] A. Al Elaiw, S. Tayachi, Different asymptotic behavior of global solutions for a parabolic system with nonlinear gradient terms. J. Math. Anal. Appl, 387 (2012), 970-992.

[7] L. Amour, M. Ben-Artzi, Global existence and decay for viscous Hamilton-Jacobi equations. J. Nonlinear Anal, 31 (1998), no. 5-6, 621-628.

[8] A. Attar, R. Bentifour, Existence of positive solutions to nonlinear elliptic systems involving gradient term and reaction potential. Electron. J. Differential Equations, (2017), no. 113, 1-10.

[9] P. Baras, M. Pierre, Problèmes paraboliques semi-linéaires avec données mesures. J. Appl. Analysis, 18 (1984), 111-149. 
[10] S. Benachour, S. Dabuleanu, The mixed Cauchy-Dirichlet problem for a viscous Hamilton-Jacobi equation. Adv. Differential Equations, 8 (2003), no. 12, 1409-1452.

[11] S. Benachour, S. Dabuleanu-Hapca, Ph. Laurençot, Decay estimates for a viscous Hamilton-Jacobi equation with homogeneous Dirichlet boundary conditions. Asymptot. Anal, 51 (2007), no. 3-4, 209-229.

[12] S. Benachour, Ph. Laurençot, Global solutions to viscous Hamilton-Jacobi equation with irregular intial data. J. Comm. Partial differential Equations, 24 (1999), 1999-2021.

[13] M. Ben-Artzi, Global existence and decay for a nonlinear parabolic equation. J. Nonlinear Anal. 19 (1992), no. 8, 763-768

[14] M. Ben-Artzi, Ph. Souplet, F. B. Weissler, The local theory for the viscous Hamilton-Jacobi equations in Lebesgue spaces. J. Math. Pures Appl, 81 (2002), 343-378.

[15] Ph. Bénilan, L. Boccardo, T. Gallouët, R. Gariepy, M. Pierre, J.L Vázquez, An L $L^{1}$-theory of existence and uniqueness of solutions of nonlinear elliptic equations. J. Ann. Scuola Norm. Sup. Pisa Cl. Sci, (4) 22 (1995), no. 2, 241-273.

[16] A. Bensoussan, J. Frehse, Smooth solutions of system of quasilinear parabolic equations, ESAIM: Control, Optimisation and Calculus of Variations, 8 (2002), 169-193.

[17] D. Blanchard, F. Murat, Renormalised solutions of nonlinear parabolic problems with $L^{1}$ data: existence and uniqueness. Proc. Roy. Soc. Edinburgh, Sect. A, 127 (1997), no. 6, 1137-1152.

[18] L. Boccardo, A. Dall'Aglio, T. Gallouët, L. Orsina, Nonlinear parabolic equations with measure data. J. Functional Analysis. 147 (1997), 237-258.

[19] L. Boccardo, L. Orsina, A. Porretta, Existence of finite energy solutions for elliptic systems with $L^{1}-$ value nonlinearities. Mathematical Models and Methods in Applied Sciences, 18 (2008), no. 5, 669-687.

[20] L. Boccardo, L. Orsina, J.-P. Puel, A quasilinear elliptic system with natural growth terms. Annali di Matematica, 194 (2015), no. 3, 1733-750.

[21] N. Boudiba, Existence globale pour des systèmes de reaction-diffusion avec controle de masse. Thèse de doctorat. Université de Rennes 1, France (1999).

[22] L. Caffarelli, R. Kohn, L. Nirenberg, First order interpolation inequalities with weights. Compositio Math, 53 (1984), 259-275.

[23] S. Clain, J. Rappaz, M. Swierkosz, R. Touzani, Numerical modeling of induction heating for two-dimensional geometries. Math. Models Methods Appl. Sci, 3 (1993), no. 6, 805-822.

[24] M.G. Crandall, P.L. Lions, P.E. Souganidis, Maximal solutions and universal bounds for some partial differential equations of evolution. Arch. Rational Mech. Anal, 105 (1989), no. 2, 163-190.

[25] Z. Dahmani, S. Kerbal, Solution to nonlinear gradient depending systems with a balance law, Electron. J. Diff. Equ. (2007), No.158, 7p.

[26] A. Dall'Aglio, D. Giachetti, C. Leone, S. Segura, Quasi-linear parabolic equations with degenerate coercivity having a quadratic gradient term. Ann. Inst. H. Poincaré Anal. non Linéaire, 23 (2006), 97-126.

[27] A. Dall'Aglio, D. Giachetti and S. Segura Semilinear parabolic equations with superlinear reaction terms, and application to some convection-diffusion problems. Ukr. Math. Bull, 1 (2004), 518-531.

[28] J. I. Diaz, M. Lazzo, P. G. Schmidt, Large solutions for a system of elliptic equation arising from fluid dynamics. Siam Journal on Mathematical Analysis, 37 (2005), 490-513.

[29] J. I. Diaz, J.-M. Rakotoson, P. G. Schmidt, A parabolic system involving a quadratic gradient term related to the Boussinesq approximation. Rev. R. Acad. Cien. Serie A. Mat. 101 (2007), no. 1, 113-118.

[30] J. I. Diaz, J.-M. Rakotoson, P. G. Schmidt, Local strong solutions of a parabolic system related to the Boussinesq approximation for buoyancy-driven flow with viscous heating. Adv. Differential Equations, 13 (2008), no. 9-10, 977-1000.

[31] E. DiBenedetto, Degenerate parabolic equations. ISBN 0-387-94020-0. Springer-Verlag.

[32] M. Escobedo, M. A. Herrero, Boundedness and blow up for a semi-linear reaction-diffusion system. J. Differential Equations 89 (1991), no. 1, 176-202.

[33] J. R. Ferguson, J. M. Fiard, R. Herbin, A two dimensional simulation of a solid oxide fuel cell. International energy agency worshop : Fundamental barries of SOFC performence, Lausane, Switzerland, August 1992.

[34] T. Gallouët, R. Herbin, Existence of solution to a coupled elliptic system. Applied. Math. Lett, 7 (1994), no. $2,49-55$.

[35] B. H. Gilding, M. Guedda, R. Kersner, The Cauchy problem for $u_{t}=\Delta u+|\nabla u|^{q}$. J. Math. Anal. Appl. 284 (2003), no. 2, 733-755. 
[36] N. Igbida, M. Kirane, Blow up for a completely coupled Fujita type reaction-diffusion system. Colloq. Math. 92 (2002), no. 1, 87-96.

[37] M. Kirane, S. Kouachi, Said Global solutions to a system of strongly coupled reaction-diffusion equations. Nonlinear Anal. 26 (1996), no. 8, 1387-1396.

[38] K Hansson, V.G. Maz'ya, I.E. Verbitsky, Criteria of solvability for multidimensional Riccati equations, Ark. Mat., 37, (1999), 87-120.

[39] J.-L. Lions, Quelques méthodes de résolution des problèmes aux limites non linéaires. (French) Dunod; Gauthier-Villars, Paris 1969.

[40] M. Marras, S. Vernier Piro, G. Viglialoro, Estimates from below of blow-up time in a parabolic system with gradient term . International Journal of Pure and Applied Mathematics, 93 (2014), no. 2, 297-306.

[41] N. C. Phuc, Morrey global bounds and quasilinear Riccarti type equation bellow the natural exponent. J. Math. Pures Appl, (9) 102 (2014), no. 1, 99-123.

[42] A. Prignet, Existence and uniqueness of "entropy" solutions of parabolic problems with $L^{1}$ data, J. Nonlinear Anal. 28 (1997), no. 12, 1943-1954.

[43] A. Porretta, Ph. Souplet, Blow-up and regularization rates, loss and recovery of boundary conditions for the super quadratic viscous Hamilton-Jacobi equation. J. Math. Pures Appl. //doi.org/10.1016/j.matpur.2019.02.014.

[44] P. Quittner, Ph. Souplet, Super linear Parabolic Problems, Blow-up, Global Existence and Steady States. ISBN 978-3-7643-8441-8. Springer-Verlag.

[45] J. Nečas, Sur une méthode pour résoudre les équations aux dérivées partielles du type elliptique, voisine de la variationelle, Ann. Scuola Norm. Sup. Pisa Ser. 16 (1962) 305-326.

[46] P. Shi, Q. Zhang, Global solutions and self-similar solutions of semilinear parabolic equations with nonlinear gradient terms, Nonlinear Anal., Theory Methods Appl., Ser. A, v.72(6), (2010), 2744-2752.

[47] Ph. Souplet, Recent results and open problems on parabolic equations with gradient nonlinearities. Electron. J. Differential Equations, (2001), no. 10, 19 pp.

[48] Ph. Souplet, Gradient blow-up for multidimensional nonlinear parabolic equations with general boundary conditions. Differential Integral Equations 15 (2002), 237-256.

[49] Ph. Souplet, F. B. Weissler, Poincaré's inequality and global solutions for a nonlinear parabolic equation. Ann. Inst. H. Poincaré, Anal. Non Linéaire, 16 (3) (1999), 335-371.

[50] Ph. Souplet, Q. S. Zhang, Global solutions of inhomogeneous Hamilton-Jacobi equations. J. Anal. Math, 99 (2006), 355-396.

[51] T.T. Tchamba, Large time behavior of solutions of viscous Hamilton-Jacobi equations with superquadratic Hamiltonian. Asymptot. Anal, 66 (2010), no. 3-4, 161-186.

[52] Al.Tersenov, Ar.Tersenov, The Cauchy problem for a class of quasilinear parabolic equations, Ann. Mat. Pura Appl., v. 182(3), (2003), 325-336.

[53] M.Wiegner, Global solutions to a class of strongly coupled parabolic systems, Math. Ann., v.292(4), (1992), 711-727.

B. Abdellaoui, A. Attar, R. Bentifour, Laboratoire d’Analyse nonlinéaire et Mathématiques ApPLIQUÉES.

Département de Mathématiques, Université Abou Bakr Belkaïd, Tlemcen,

Tlemcen 13000, Algeria.

E.-H. Laamri, Institut Elie Cartan,

UNIVERSITÉ LORRAINE,

B. P. 239, 54506 VAndeuvre lés NAnCy, France.

E-mail addresses:

boumediene.abdellaoui@inv.uam.es, ahm.attar@yahoo.fr,

rachidbentifour@gmail.com, el-haj.laamri@univ-lorraine.fr. 